\title{
Effects of Selective Deafferentation on the Discharge Characteristics of Medial Rectus Motoneurons
}

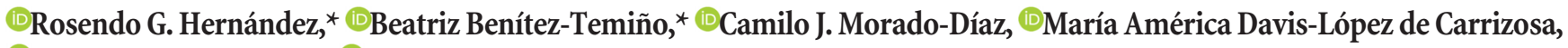 \\ - Rosa R. de la Cruz, and ${ }^{\circ}$ Angel M. Pastor \\ Departamento Fisiología, Facultad de Biología, Universidad de Sevilla, 41012 Sevilla, Spain
}

Medial rectus motoneurons receive two main pontine inputs: abducens internuclear neurons, whose axons course through the medial longitudinal fasciculus (MLF), and neurons in the lateral vestibular nucleus, whose axons project through the ascending tract of Deiters (ATD). Abducens internuclear neurons are responsible for conjugate gaze in the horizontal plane, whereas ATD neurons provide medial rectus motoneurons with a vestibular input comprising mainly head velocity. To reveal the relative contribution of each input to the oculomotor physiology, single-unit recordings from medial rectus motoneurons were obtained in the control situation and after selective deafferentation from cats with unilateral transection of either the MLF or the ATD. Both MLF and ATD transection produced similar short-term alterations in medial rectus motoneuron firing pattern, which were more drastic in MLF of animals. However, long-term recordings revealed important differences between the two types of lesion. Thus, while the effects of the MLF section were permanent, 2 months after ATD lesioning all motoneuronal firing parameters were similar to the control. These findings indicated a more relevant role of the MLF pathway in driving motoneuronal firing and evidenced compensatory mechanisms following the ATD lesion. Confocal immunocytochemistry revealed that MLF transection produced also a higher loss of synaptic boutons, mainly at the dendritic level. Moreover, 2 months after ATD transection, we observed an increase in synaptic coverage around motoneuron cell bodies compared with short-term data, which is indicative of a synaptogenic compensatory mechanism of the abducens internuclear pathway that could lead to the observed firing and morphological recovery.

Key words: lesion-induced plasticity; motoneuron; oculomotor system

Significance Statement

Eye movements rely on multiple neuronal circuits for appropriate performance. The abducens internuclear pathway through the medial longitudinal fascicle (MLF) and the vestibular neurons through the ascending tract of Deiters (ATD) are a dual system that supports the firing of medial rectus motoneurons. We report the effect of sectioning the MLF or the ATD pathway on the firing of medial rectus motoneurons, as well as the plastic mechanisms by which one input compensates for the lack of the other. This work shows that while the effects of MLF transection are permanent, the ATD section produces transitory effects. A mechanism based on axonal sprouting and occupancy of the vacant synaptic space due to deafferentation is the base for the mechanism of compensation on the medial rectus motoneuron.

\section{Introduction}

Lateral and medial rectus extraocular muscles constitute the agonist/antagonistic pair involved in the generation of horizontal

\footnotetext{
Received May 22, 2017; revised July 11, 2017; accepted Aug. 6, 2017.

Author contributions: B.B.-T., R.R.d.I.C., and A.M.P. designed research; R.G.H., B.B.-T., C.J.M.-D., and A.M.P. performed research; R.G.H. and M.A.D.-L.d.C. analyzed data; B.B.-T., R.R.d.I.C., and A.M.P. wrote the paper.

This work was supported by Ministerio de Ciencia e Innovacion-Fondo Europeo de Desarrollo Regional Grants BFU2015-64515-P and BFU2012-33975. R.G.H. was a fellowship holder of the Universidad de Sevilla. Imaging studies were performed at Centro de Investigación Tecnología e Innovación de la Universidad de Sevilla. *R.G.H. and B.B.-T. are co-first authors.

The authors declare no competing financial interests.

Correspondence should be addressed to Angel M. Pastor, Departamento de Fisiología, Universidad de Sevilla, Avenida Reina Mercedes 6, 41012 Sevilla, Spain. E-mail: ampastor@us.es.
}

gaze. Their parent motoneurons lie in the abducens and oculomotor nuclei, respectively (Büttner-Ennever, 2006). Both types of motoneurons display a similar tonic-phasic discharge pattern during eye fixations and saccades, respectively (Delgado-Garcia et al., 1986a; Fuchs et al., 1988; de la Cruz et al., 1989; Pastor et al., 1991). Despite their comparable firing pattern and parallel function in conjugate eye movements, the synaptic organization of lateral rectus and medial rectus motoneurons is quite different. Thus, abducens motoneurons receive direct afferents mainly from a threefold system that conveys signals associated to 
saccades (pontomedullary reticular formation), slow phases of the vestibular nystagmus (medial vestibular nucleus), or eye fixations (Hikosaka et al., 1978; Grantyn et al., 1980; Igusa et al., 1980; McCrea et al., 1980; McCrea and Baker, 1985; Escudero and Delgado-Garcia, 1988; Büttner-Ennever, 2006; prepositus hypoglossi nuclei).

On the contrary, the main input to medial rectus motoneurons conveys an already integrated signal originated at the abducens internuclear neurons, which project contralaterally through the medial longitudinal fascicle (MLF) and mediate horizontal conjugate gaze (Highstein and Baker, 1978; Carpenter and Carleton, 1983; McCrea et al., 1986; Delgado-Garcia et al., 1986b; González-Forero et al., 2005; Büttner-Ennever, 2006). The importance of this pathway has been revealed in lesion experiments where the MLF has been sectioned, inducing the syndrome of internuclear ophthalmoplegia, which involves the disturbance of versional horizontal eye movements, the abduction position of the ipsilateral eye at rest, and the inability of this eye to cross into the nasal visual hemifield, except during convergence movements (Carpenter and McMasters, 1963; Highstein, 1977; Highstein and Baker, 1978; de la Cruz et al., 2000).

The second source of pontine afferences to medial rectus motoneurons arises in the ventral portion of the lateral vestibular nucleus and projects ipsilaterally through the ascending tract of Deiters (ATD), which courses lateral to the MLF (Baker and Highstein, 1978; Furuya and Markham, 1981; Büttner-Ennever, 2006). The role of this input has been interpreted as redundant since it encodes head velocity (HV) and eye position (EP) signals that are also carried by abducens internuclear neurons (Reisine and Highstein, 1979; Reisine et al., 1981). However, there are striking differences between MLF and ATD pathways concerning the strategic somatodendritic distribution of their respective synaptic terminals. In particular, ATD neurons terminate preferentially over the soma and proximal dendrites of medial rectus motoneurons, whereas the majority of abducens internuclear synaptic endings contact distal dendrites (Nguyen et al., 1999), therefore pointing to a crucial role of the ATD pathway in the processing of the information coming from abducens internuclear neurons (Nguyen et al., 1999).

In the present work, we have performed the unilateral section of either the MLF or the ATD to reveal the relative influence of these two inputs. For this purpose, we have recorded in chronically alert cats the discharge of partially deafferented medial rectus motoneurons, simultaneously with eye movements. We were also interested in determining whether there was some type of recovery in motoneuronal firing with time following the selective lesion of each input, and in evaluating the possibility of compensatory mechanisms that could be developed by the pathway that was left intact. We demonstrate the functional and morphological prevalence of the MLF to drive motoneuronal firing and to compensate for the lesion of the ATD, likely through axonal sprouting. On the contrary, the ATD pathway was not able to compensate for the more drastic effects of the MLF lesion.

\section{Materials and Methods}

Experimental design and statistical analysis. Experiments were performed in a total of 15 adult female cats weighing $2.5-3.5 \mathrm{~kg}$ that were obtained from authorized breeders (University of Córdoba, Córdoba, Spain). Seven animals used for chronic recordings were divided into two groups to carry out the section of either the MLF $(n=3)$ or the ATD $(n=4)$. Four different animals were used for morphological analysis $3 \mathrm{~d}$ after $\operatorname{ATD}(n=2)$ or MLF $(n=2)$ transection [namely, short term (st)]. Four control animals were used for the anterograde tracing with biocytin from either the contralateral abducens $(n=2)$ or the ipsilateral lateral vestibular nucleus $(n=2)$. All experimental procedures were in compliance with the European Union Directive (2010/63/EU) on the protection of animals used for scientific purposes and Spanish legislation (R.D. 55/2013).

Data were expressed as the mean \pm SEM. Comparisons between electrophysiological and morphological data obtained from MLF- or ATD-sectioned animals and controls at different times postlesion were performed using the two-way ANOVA test, with an overall level of significance of 0.05 , followed by post hoc comparisons using the HolmSidak test. All regression lines obtained in the present work were significant $(p<0.05)$. Statistical analysis was performed with the aid of SigmaPlot software, version 11.0 (SYSTAT).

Procedures for chronic recordings. Animals were protected from vagal reflexes by means of atropine sulfate $(0.5 \mathrm{mg} / \mathrm{kg}$, i.m. $)$, anesthetized with ketamine hydrochloride $(20 \mathrm{mg} / \mathrm{kg}$, i.m. $)$ mixed with xylazine $(0.5 \mathrm{mg} / \mathrm{kg}$, i.m.) and then placed in a stereotaxic frame. Under aseptic conditions, stimulating electrodes, scleral coils, and the recording chamber were implanted. For the antidromic identification of the oculomotor nucleus, silver bipolar electrodes were implanted in the third nerve bilaterally. Additionally, a silver bipolar electrode was also implanted in the right sixth nerve at its exit from the brainstem for antidromic abducens nucleus identification. Two-turn Teflon-insulated stainless steel eye coils were implanted in the sclera of both eyes for eye movement recordings. Finally, two square-shaped windows were drilled in the skull to allow access both to the oculomotor nucleus in the midbrain, through the parietal cortex, and to the abducens nucleus in the pons, through the cerebellum. Acrylic chambers were built around the windows and bolted to the cranium for the following two purposes: first, to keep aseptic conditions; and second, as a part of a restraining system to prevent head movements during recordings. Animals were allowed to recover for $10-15 \mathrm{~d}$. During this period, animal care was taken to ensure the healthy state of the animal.

Chronic recordings, analysis, and lesion procedures. Recording sessions were performed every $2 \mathrm{~d}$ except from the first week after axotomy, when recording sessions occurred daily. During the recording sessions, animals were comfortably seated in a fabric bag, bandaged, and placed in a Plexiglas box inside a coil frame that was situated on a servo-controlled table to evoke the vestibulo-ocular reflex in cats. Eye movements were recorded using the magnetic field search-coil technique (Fuchs and Robinson, 1966).

Extracellular recordings in the medial rectus subdivision of the oculomotor nucleus were performed with beveled glass micropipettes and filled with $2 \mathrm{M} \mathrm{NaCl}$. Medial rectus subdivision of the oculomotor nucleus was located stereotaxically and with the aid of the antidromic field potential produced by electrical stimulation of the third nerve. Medial rectus motoneurons were identified by the following three different criteria: first, its preferential dorsolateral position in the nucleus; second, the increase in firing frequency during nasally directed horizontal eye movements; third, the collision test between the antidromic and the orthodromic action potentials. The extracellular neuronal activity was amplified and filtered at a bandwidth of $10 \mathrm{~Hz}$ to $10 \mathrm{kHz}$ for display and digitalization purposes.

Eye and head movement and unitary neuronal recordings were stored for off-line analysis using a digitizing card (Power 1401, Cambridge Electronic Design). The range of eye movements was measured in controls and at different times postlesion, in both ATD- and MLF-sectioned animals, by selecting at least 21 periods of $100 \mathrm{~s}$ of spontaneous eye movements $(n \geq 21$ and $n \leq 135)$ for each lesion type and time point. The analysis of motoneuron firing rate (FR) was performed following the protocol previously described (Davis-López de Carrizosa et al., 2009). Briefly, data for EP, eye velocity (EV), HV, and neuronal instantaneous FR were selected by means of custom-made programs written in MATLAB version 7.5. The firing rate of medial rectus motoneurons can be fitted according to the equation $\mathrm{FR}=F_{0}+k \cdot \mathrm{EP}+r \cdot \mathrm{EV}$, where the coefficients $k$ (in spikes/s/degree) and $r$ (in spikes/s/degree/s) are the neuronal sensitivities to $\mathrm{EP}$ and $\mathrm{EV}$, respectively, and $F_{0}$ is the ordinate intercept. During eye fixations, eye velocity is zero, and, therefore, the firing rate was fitted to the equation $\mathrm{FR}=k \cdot \mathrm{EP}+F_{0}$. Relationships 
between neuronal firing and EV (in degrees/s) during saccades were obtained by linear regression analysis after subtraction of the position component $(k \cdot \mathrm{EP})$, which was calculated from the previously known sensitivity to eye position. During spontaneous eye movements, we named these two coefficients as $k_{s}$ and $r_{s}$. Mean $k_{s}$ and $r_{s}$ values were obtained for each experimental group at every time point $(n \geq 13$ and $n \leq 98 ; n=$ number of cells recorded and analyzed per group).

We also recorded eye and head position and velocity during the performance of the vestibulo-ocular reflex, elicited by rotating the table (and thus the animal's head) at known angles in the horizontal plane in darkness. We measured the gain of the reflex during a minimum of 20 intervals of $100 \mathrm{~s}$ ( $n \geq 20$ and $n \leq 51$ ) for each experimental group and the velocity of the fast phases of the nystagmus for the different experimental situations ( $n \geq 42$ and $n \leq 339 ; n=$ reflex hemicycles analyzed for fast phases).

Motoneuron firing rate during vestibularly induced eye movements also followed the above-described equation, but coefficients were named $k_{v}$ and $r_{v}$. Moreover, we also analyzed separately the firing rate during on-directed and off-directed slow phases of the reflex. Mean values for $k_{v}$, $k_{v} \mathrm{ON}, k_{v} \mathrm{OFF}, r_{v}, r_{v} \mathrm{ON}$, and $r_{v}$ OFF were calculated and compared statistically ( $n \geq 11$ and $n \leq 56 ; n=$ number of cells analyzed for each experimental group and time point). Due to the motor impairment of the eye ipsilateral to the fascicle transection, we used the eye movement of the contralateral eye for computations (de la Cruz et al., 2000). Recordings were performed for a period of 2 months after lesioning. Data were grouped in $0-5,6-15$, and $16-25 \mathrm{~d}$ after lesion [namely, st, 15 , and $25 \mathrm{~d}$ after lesion, respectively] and between $25 \mathrm{~d}$ and 2 months after lesion [namely, long term (lt)].

After an approximate recording period of 2 weeks in which control recordings were performed for each animal, the lesion was created with a custom-made microblade driven by a Canberra-type micromanipulator with an anteriorly directed angle of $45^{\circ}$ to reach either the MLF or the ATD caudal to the trochlear nucleus. The lesion tract was guided by stereotaxic coordinates and by the recordings of the abducens nucleus and the MLF.

Retrograde medial rectus motoneuron labeling. Seven days before the end of the experiment, medial rectus muscles were injected bilaterally with rhodamine isothiocyanate (Sigma-Aldrich) for motoneuron retrograde identification. Under general anesthesia and with the help of a dissecting microscope, the medial rectus muscle of each eye was exposed and a volume of $8 \mu \mathrm{l}$ of $20 \%$ rhodamine prepared in a solution of $2 \%$ dimethylsulfoxide was injected using a Hamilton syringe.

Immunocytochemistry. Animals were deeply anesthetized (sodium pentobarbital, $50 \mathrm{mg} / \mathrm{kg}$, i.p.), and immediately perfused transcardially with saline followed by $4 \%$ paraformaldehyde prepared in phosphate buffer $0.1 \mathrm{M}$, pH 7.4. The 50- $\mu \mathrm{m}$-thick coronal sections in the brainstem were obtained using a vibratome and collected in a solution of PBS, $\mathrm{pH} 7.4$, with $0.05 \%$ sodium azide for proper storage at $4^{\circ} \mathrm{C}$. Selected sections were stained following the Nissl method to assess ATD and MLF lesions.

To assess the presence of calretinin in vestibular neurons, as well as in the MLF and ATD, and to check the correct localization of the lesion site, brainstem sections were selected rostral to abducens and caudal to the trochlear nucleus. The proximal stumps of sectioned axons were identified by immunocytochemistry against calretinin (goat anti-calretinin; 1:2500; AB1550, Millipore Bioscience Research Reagents), a calciumbinding protein that is expressed by both abducens internuclear neurons and neurons located at the ventral portion of the lateral vestibular nucleus (de la Cruz et al., 1998; Highstein and Holstein, 2006). Briefly, tissue was blocked with $7 \%$ normal donkey serum (NDS) in PBS with $0.1 \%$ Triton X-100 (PBS-T) for $45 \mathrm{~min}$, and incubated overnight at room temperature in a solution containing the primary antibody in PBS-T with $0.05 \%$ sodium azide and 3\% NDS. After several washes, slices were incubated for $90 \mathrm{~min}$ in the secondary antibody (1:500 in PBS-T; biotinylated donkey anti-goat IgG, Vector Laboratories). Sections were then rinsed and incubated for $90 \mathrm{~min}$ in the avidin-biotin-HRP complex (ABC, Vector Laboratories) prepared in PBS-T. After sequential washes in PBS, Tris-HCl-buffered saline, $\mathrm{pH}$ 7.4, Tris buffer $0.1 \mathrm{M}, \mathrm{pH} 7.4$, and Tris buffer $0.1 \mathrm{M}, \mathrm{pH} 8$, tissue was incubated in a solution of $0.05 \% 3,3^{\prime}$ diaminobenzidine tetrahydrochloride as the chromogen, $0.01 \%$ hydrogen peroxide, and nickel sulfate $1 \mathrm{~mm}$ prepared in Tris buffer $0.1 \mathrm{M}, \mathrm{pH} 8$.
Sections were thoroughly washed, mounted on glass slides and coverslipped with DPX (Sigma-Aldrich). All animals showed the complete transection of the selected fascicle, either the MLF or the ATD.

In addition, we performed immunocytochemistry against different antibodies in midbrain sections obtained from animals sectioned at the $\operatorname{ATD}$ ( $n=4$ for the long-term study; $n=2$ for the short-term study) or at the MLF ( $n=3$ for the long-term study; $n=2$ for the short-term study). For the morphological study, long term was 2 months and short term was $3 \mathrm{~d}$. First, to characterize the synaptic organization at the medial rectus motoneuron subdivision of the oculomotor nucleus, we used double immunofluorescence directed against calretinin to evaluate MLF or ATD innervation, along with synaptophysin (mouse anti-Syn; 1:2000; MAB329, Millipore), a general marker of synaptic boutons. After several washes in PBS, midbrain sections were first incubated for 10 min in PBS with $1 \%$ sodium borohydride to increase antibody penetration, blocked as described above, and incubated for $12 \mathrm{~h}$ at room temperature in a solution containing the primary antibody in PBS-T with $0.05 \%$ sodium azide and 3\% NDS. After washing, sections were incubated for $2 \mathrm{~h}$ in the secondary antibody, prepared in PBS-T [1:50; donkey anti-mouse IgG combined with fluorescein isothiocyanate (FITC) and donkey anti-goat IgG coupled to Cy5 for synaptophysin and calretinin visualization, respectively; Jackson Immunoresearch]. After rinsing, sections were mounted on glass slides and coverslipped with VECTASHIELD (Vector Laboratories).

Second, we performed immunocytochemistry against GAP-43 (mouse anti-GAP-43, 1:1000; MAB347, Millipore) to visualize axonal sprouting. Briefly, sections were blocked as described above and incubated overnight at room temperature in PBS-T containing the primary antibody. After several rinses in PBS-T, sections were immersed in a solution containing the secondary antibody (1:50; donkey anti-mouse IgG combined with FITC; Jackson ImmunoResearch).

Finally, some sections were selected to assess the possibility of changes in neurotrophin expression in partially deafferented medial rectus motoneurons. For this purpose, we performed immunocytochemistry against brain-derived neurotrophic factor (BDNF), neurotrophin-3 (NT-3), or nerve growth factor (NGF). Sections were incubated for 10 min in PBS with $1 \%$ sodium borohydride, blocked as described above, and incubated for $12 \mathrm{~h}$ at room temperature in a solution containing the primary antibody in PBS-T with $0.05 \%$ sodium azide and 3\% NDS (1:100; rabbit anti-BDNF; sc-546; rabbit anti-NT3, sc-547; or rabbit anti-NGF, sc-548; all Santa Cruz Biotechnology). After several washes, sections were incubated first in the secondary antibody (1:250; biotinylated donkey antirabbit, Vector Laboratories) for $2 \mathrm{~h}$ and then in streptavidin conjugated with Cy2 (1:800 in PBS, 45 min; Jackson ImmunoResearch).

Anterograde abducens/vestibular axon labeling. Four control animals were selected to track the course and final arborization of either abducens $(n=2)$ or vestibular $(n=2)$ axons toward medial rectus motoneurons. Briefly, biocytin (3\% in Tris- $\mathrm{HCl}$ buffer $0.05 \mathrm{M}, \mathrm{pH}$ 7.4; Sigma-Aldrich) was injected unilaterally using a micropipette, stereotaxically guided toward either the abducens nucleus or the lateral vestibular nucleus. Cathodal positive pulses of $7 \mu \mathrm{A}$ and $80 \%$ duty cycle were applied for $15 \mathrm{~min}$. Twenty-four hours later, animals were deeply anesthetized (sodium pentobarbital, $50 \mathrm{mg} / \mathrm{kg}$, i.p.) and perfused intracardially with physiological saline followed by the fixative containing 3\% paraformaldehyde and $1.25 \%$ glutaraldehyde in $0.1 \mathrm{~m}$ sodium phosphate buffer, $\mathrm{pH}$ 7.4. The $50-\mu \mathrm{m}$-thick coronal sections were revealed using the $\mathrm{ABC}$ method (Vector Laboratories), prepared in PBS-T, and treated as described above to reveal the peroxidase reaction. Selected sections were counterstained with $0.5 \%$ toluidine blue for Nissl staining.

Image analysis. In the anterograde labeling experiments, brainstem sections were observed and images were captured using a Zeiss optical conventional microscope connected to a camera (AxioCam ERc 5s, Zeiss) and a computer for image storage and analysis. Control sections were used to count the number of calretinin-positive axonal processes located inside the limits of the MLF or the ATD per slice. Mean and SEM were calculated to compare the extension of each projection on medial rectus motoneurons.

Midbrain immunostained sections were observed, and images were captured using a Zeiss LSM Duo Confocal Microscope (Zeiss). Lasers 
DPSS $561 \mathrm{~nm}$, argon $488 \mathrm{~nm}$, and HeNe $633 \mathrm{~nm}$ were used to excite rhodamine, FITC or Cy2, and Cy5, respectively. For the analysis of synaptic coverage around medial rectus motoneuron somata, images of a single focal plane were made using the $63 \times$ oil-immersion objective. Using ImageJ software (National Institutes of Health), the number of axon terminals per soma was counted, and the somatic coverage was calculated as the percentage of the soma perimeter that was occupied by synaptophysin- or calretinin-immunoreactive boutons ( $n \geq 32$ and $n \leq$ $268 ; n=$ number of cells analyzed). For the analysis of synaptic density in the neuropil and GAP-43 expression, we used the oil-immersion $40 \times$ objective. Random-selected squares of $30.5 \times 30.5 \mu \mathrm{m}$ through the neuropil were used to measure optical density. Background level was subtracted, and the values of optical density were normalized with respect to the control side of the same section $(n \geq 210, n \leq 1300 ; n=$ number of squares selected per experimental group and time point). For the analysis of neurotrophin expression, optical density was measured inside the cytoplasm of control and deafferented medial rectus motoneurons of the same section. After subtraction of the background level, data were expressed for each cell as the percentage with respect to the mean of the control values in the same section $(n \geq 22, n \leq 161$ for BDNF; $n \geq 24$ and $n \leq 138$ for NT-3; $n \geq 17$ and $n \leq 169$ for NGF; $n=$ number of cells selected per experimental group and time point).

\section{Results}

Comparison of abducens and vestibular inputs to medial rectus motoneurons

Anterograde transport of biocytin, injected either into the abducens nucleus (Fig. 1A, asterisk) or the lateral vestibular nucleus (Fig. $1 B$, asterisk) of control animals, allowed the tracing of both neuronal afferences to medial rectus motoneurons. As previously described (Bienfang, 1978), axons from abducens internuclear neurons cross the midline at the level of the abducens nucleus and project rostrally through the contralateral MLF (Fig. 1C), a bundle of axons that course the brainstem longitudinally, in a dorsomedial position. Abducens projection onto medial rectus motoneurons was densely packed at the medial rectus subdivision of the oculomotor nucleus (Fig. $1 E$, arrows). On the other hand, as described by Baker and Highstein (1978), vestibular afferences to medial rectus motoneurons project through the ipsilateral ATD, a fascicle located immediately lateral to the MLF (Fig. 1D), and reach the oculomotor nucleus, forming a much smaller and less branched terminal net of axons when compared with that of abducens internuclear neurons (Fig. $1 F$, arrows). Axonal quantification revealed a fourfold higher number of labeled axons in the MLF than in the ATD (818.25 \pm 26.82 and $243.25 \pm 34.67$ positive axons/section in MLF and ATD, respectively; mean \pm SEM, $n=4, t$ test; $\left.t_{(6)}=13.11 ; p<0.001\right)$. Higher-magnification images showed that abducens internuclear neurons (Fig. 1G) and vestibular axon terminals (Fig. $1 J$ ) formed bouton-like varicosities. However, most abducens internuclear neuron terminals were found intermingled in the neuropil of the oculomotor nucleus (Fig. $1 \mathrm{H}, \mathrm{I}$ ), while vestibular afferences were found more frequently contacting medial rectus motoneuron cell bodies (Fig. $1 K, L$ ), in accordance with the results of the study by Nguyen et al. (1999).

\section{Vestibular projection to medial rectus motoneurons is immunopositive against calretinin}

Previous immunocytochemistry studies have shown that cat abducens internuclear and vestibular neurons express the calciumbinding protein calretinin (de la Cruz et al., 1998; Baizer and Baker, 2005). However, a demonstration of the presence of calretinin-positive axons in the ATD is still lacking. Figure $2 \mathrm{~A}$ shows a coronal section through the pons, immunostained against calretinin. As expected, abducens internuclear neurons were positive for calretinin staining (area surrounded by the dashed line in Fig. 2A) but also for the medial vestibular nucleus, which projects to the abducens nucleus, and the lateral vestibular neurons in the area of origin of the ATD (Fig. $2 A$, inset). These results were in agreement with those obtained from more rostral sections, in which calretinin-positive axons were found both in the MLF and the ATD (Fig. 2B). In ATD-sectioned animals, positive axons were visible only in the MLF, not in the sectioned ATD (Fig. 2C). Similarly, in MLF-sectioned animals, the lack of calretinin demonstrated the extent of the lesion (Fig. 2E, asterisk). Therefore, calretinin immunostaining allowed checking of the correct location of lesions as further assessed by Nissl staining (Fig. $2 D, F)$.

\section{Changes in spontaneous eye movements after selective deafferentation}

Control unlesioned cats perform eye movements with a high degree of conjugacy. However, lack of afferent stimulation onto medial rectus motoneurons induced motor deficits, which were present from the onset of the lesion. More precisely, in the first $5 \mathrm{~d}$ after MLF transection, the eye ipsilateral to the lesion side was unable to cross toward the contralateral oculomotor hemifield (Fig. 3C). The lack of MLF input onto medial rectus motoneurons was devastating for the movement of the affected eye (Fig. $3 C$, blue trace). On the other hand, the ATD lesion also affected the ipsilateral eye but to a lesser degree and duration (Fig. $3 \mathrm{~A}$, green trace). In the following days, there was a progressive recovery of motor function in both ATD and MLF animals, but this recovery was much more pronounced in the ATD-lesioned animals (Fig. $3 B, D$ ). Spontaneous convergent eye movements could be detected throughout the entire recording period after both types of lesion. Figure 3, $E$ and $F$, illustrates the linear regression lines obtained after representing the horizontal movement of the right eye versus the left eye in the different groups. The slope of these plots (that we termed $m$ ) was close to 1 in control data (Figure $3 E, F$, black line). Short-term post-ATD lesion showed a small reduction in the range of movements of the ipsilateral eye (Fig. 3E, light green line). Interestingly, in the long term, ATD lesion-induced deficits in spontaneous eye movements disappeared and the slope returned to values similar to control (Fig. $3 E$, dark green line). In MLF animals, short-term recordings showed a severe reduction in the range of movements in the affected eye, which can be appreciated as a reduction in the $m$ value (Fig. 3F, light blue line). The amplitude of movements increased with time, but the affected eye never reached the normal range (Fig. 3F, dark blue line).

The statistical analysis revealed differences between both the two different transections and the time postlesion in the ipsilateral eye movements (two-way ANOVA, $F_{(2,3)}=4.65$; $p<0.001$, Holm-Sidak method for multiple comparisons). Thus, in the short term $(<5 \mathrm{~d}$ postlesion), the range of movements decreased after partial deafferentation, but this decrease was statistically larger $(p<0.001)$ in MLF animals $\left(19.9 \pm 0.9^{\circ}, n=\right.$ 27) than in ATD animals $\left(28.0 \pm 1.0^{\circ}, n=41\right.$; data expressed as the mean \pm SEM) when compared with controls (33.5 \pm $0.4^{\circ}, n=135$; Fig. $3 G$ ). There was a progressive recovery in the range of movements of both groups, which was more pronounced in the case of ATD animals. Thus, in the long term ( $>25 \mathrm{~d}$ postlesion) there were no statistical differences between controls and ATD animals (31.6 $\left.\pm 1.1^{\circ}, n=26\right)$, while MLF animals still exhibited lower values in the range of movements when compared with controls and long-term ATD animals $\left(26.0 \pm 0.6^{\circ}, n=29 ; p<0.001\right.$ for both comparisons; 

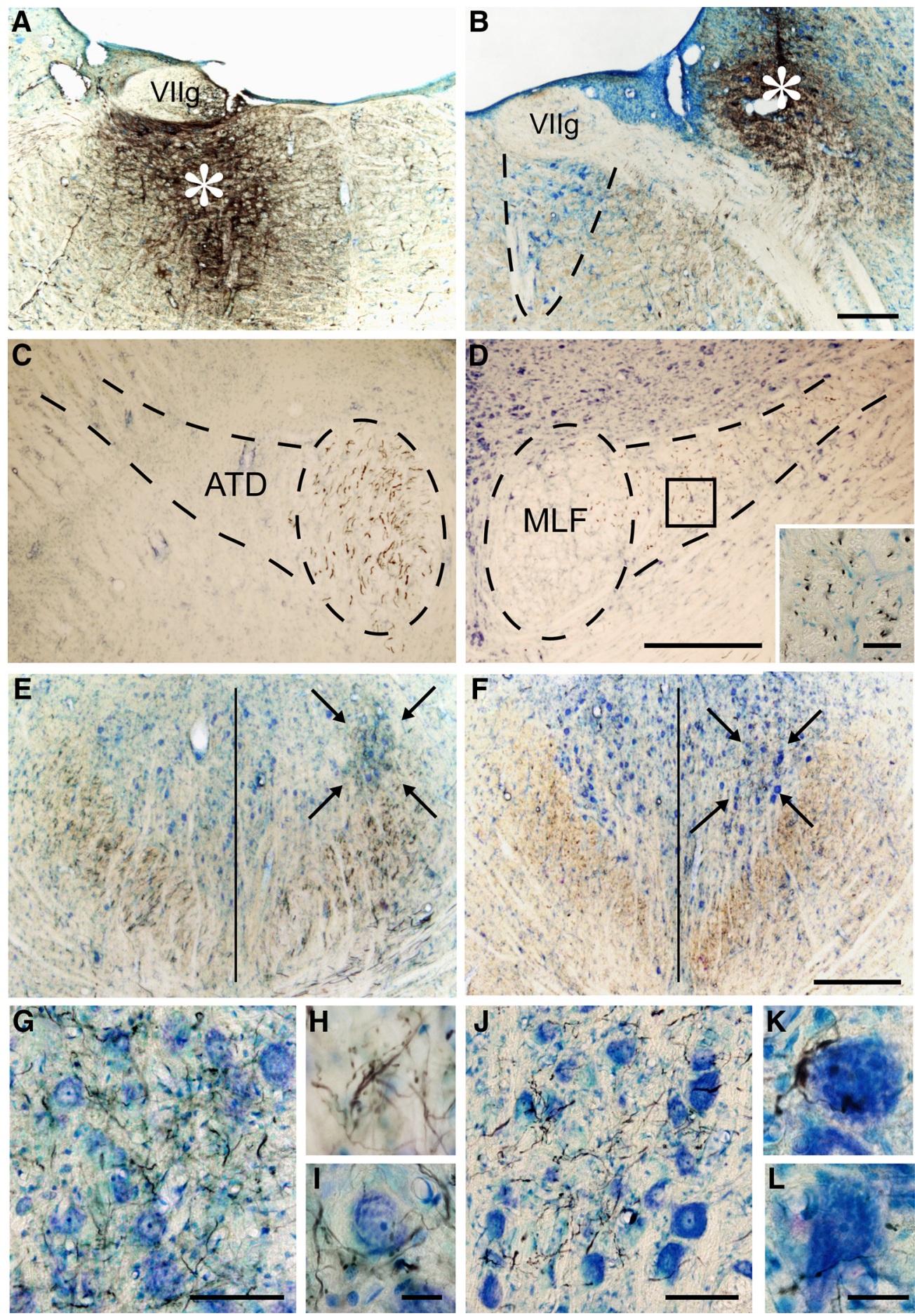

Figure 1. Anterograde tracing of pontine medial rectus motoneuron afferences. $\boldsymbol{A}, \boldsymbol{B}$, Biocytin injection (asterisks) in the abducens $(\boldsymbol{A})$ or lateral vestibular nuclei $(\boldsymbol{B})$. Vllg, Genu of the facial nerve. The dashed line marks the approximate boundaries of the abducens nucleus. $C, D$, Biocytin-labeled axons coursing through the contralateral MLF (C, for the injection shown in $A$ ) or the ipsilateral ATD $(\boldsymbol{D}$, for the injection shown in $\boldsymbol{B})$. Inset in $\boldsymbol{D}$ shows an enlarged image of the squared box in the ATD. $\boldsymbol{E}, \boldsymbol{F}$, Stained axon terminals from abducens internuclear $(\boldsymbol{E})$ or vestibular $(\boldsymbol{F})$ neurons. Arrows show the location of medial rectus subdivision. Vertical lines indicate the midline. $\mathbf{G}-\boldsymbol{L}$, Higher-magnification images obtained from the same areas delimited by arrows in $\boldsymbol{E}(\mathbf{G} \boldsymbol{I})$ and $\boldsymbol{F}(\boldsymbol{J}-\boldsymbol{L})$ showing biocytin-labeled terminals around the motoneurons. Scale bars: $\boldsymbol{B}$ (for $\boldsymbol{A}, \boldsymbol{B}), \boldsymbol{D}$ (for $\boldsymbol{C}, \boldsymbol{D}), \boldsymbol{F}$ (for $\boldsymbol{E}, \boldsymbol{F}), 500 \mu \mathrm{m} ; \boldsymbol{G}, \boldsymbol{J}, 100 \mu \mathrm{m}$; $\boldsymbol{I}$ (for $\boldsymbol{H}, \boldsymbol{I}), \boldsymbol{L}$ (for $\boldsymbol{K}, \boldsymbol{L}), 20 \mu \mathrm{m}$; inset, $100 \mu \mathrm{m}$.

Fig. $3 G)$. The statistical analysis of the $m$ value offered similar results (Fig. $3 H$ ). In control animals, the $m$ value was $0.90 \pm$ $0.01(n=135)$. During the first $5 \mathrm{~d}$ after lesioning, this value dropped to statistically lower $(p<0.001)$ values compared with controls (ATD animals: $0.70 \pm 0.03, n=41$; MLF animals, $0.55 \pm 0.03, n=27)$ that were lower in MLF animals than in ATD animals $(p<0.001)$. There was a progressive recov- ery, and in the long term ATD animals exhibited $m$ values that were similar to those of controls $(0.88 \pm 0.02, n=26)$, that were higher $(p<0.001)$ than those obtained in the short term and in MLF animals at long term $(0.74 \pm 0.03, n=29)$. The effects of MLF sectioning also improved in the long term with respect to the short term, but the recovery was incomplete and $m$ values were lower compare with those of controls at any time point (two-way ANOVA 

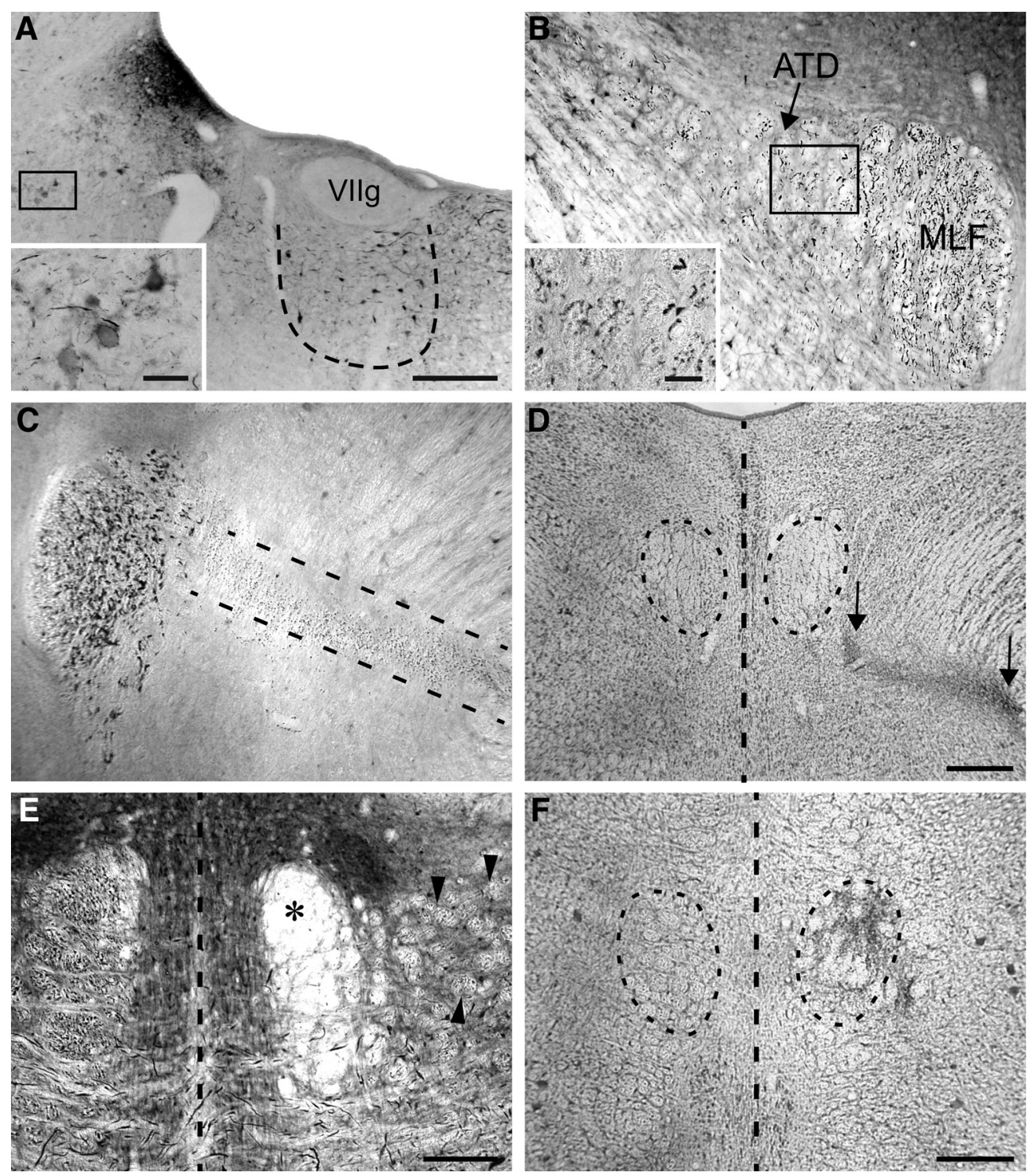

Figure 2. Lesion assessment using anti-calretinin immunostaining and Nissl labeling. $\boldsymbol{A}$, Calretinin immunolabeled both abducens internuclear neurons (dashed line) and lateral vestibular neurons (square). Inset shows a higher-magnification image of the area delimited by the square. VIlg, Facial nerve genu. $\boldsymbol{B}$, Calretinin-stained axons in both control ATD and MLF tracts. Inset magnifies the square-delimited area. $C$, Lesion site of the ATD demonstrated by the absence of calretinin and the presence of refringent scar tissue under Nomarski transillumination (dashed lines delimiting area). $\boldsymbol{D}$, Nissl demonstration of the scar tissue (delimited by arrows) corresponding to the lateral span of the ATD transection that spared the MLF (circled). $\boldsymbol{E}$, Calretinin-stained section rostral to the lesion site demonstrating lack of immunostaining (asterisk) in the severed MLF. Calretinin-stained axons were present in the ATD (arrowheads). $F$, Nissl demonstration of the unilateral lesion of the MLF (delineated by the dashed line). Midline is indicated by vertical dashed lines in $\boldsymbol{D}-\boldsymbol{F}$. Scale bars: $\boldsymbol{A}, \boldsymbol{D}, \boldsymbol{F}, 500 \mu \mathrm{m} ; \boldsymbol{E}$ (for $\boldsymbol{B}, \boldsymbol{C}, \boldsymbol{E}), 250 \mu \mathrm{m}$; insets, $\boldsymbol{A}, \boldsymbol{B}$ (for $\boldsymbol{B}, \boldsymbol{C}), 50 \mu \mathrm{m}$.

test, $F_{(2,3)}=15.65 ; p<0.001$, Holm-Sidak method for multiple comparisons).

Altogether, these results showed that vestibular input deprivation had a transient effect on horizontal motor range. In contrast, the axotomy of the abducens internuclear neuron had a more drastic effect, which, although improved with time, never reached normal values.

\section{Partial deafferentation induces eye movement deficits during the vestibulo-ocular reflex}

Sinusoidal head rotation in the horizontal plane (Fig. $3 I-L$, red lines represent the inverted head velocity) induced compensatory eye movements with approximately the same velocity but in the opposite direction (Fig. 3I- $L$, dashed black lines for control eye velocity, colored lines for the affected eye). These slow displacements were interspersed by fast phases, in the same direction as head rotation, which recentered the eye in the orbit (Fig. 3I-L). Thus, when the head moved to the left, the compensatory movements were directed to the right and the fast phases to the left. Vestibular deafferentation (ATD lesion) of medial rectus motoneurons altered the performance of the vestibulo-ocular reflex. Thus, the gain of the reflex (i.e., the ratio of eye velocity during the slow phases to head velocity) was reduced in the affected eye immediately after lesioning and during the following $5 \mathrm{~d}$ (Fig. 3I, solid green line). Moreover, the velocity of fast phases was reduced both in the on and off directions of the hemicycles of slow phases (Fig. 3I, orange and red dots, respectively). After the initial alterations, eye movements progressively recovered and were qualitatively similar to control from $15 \mathrm{~d}$ after deafferentation onward (Fig. 3J, solid green line).

On the contrary, MLF transection produced a larger and permanent reduction in both the amplitude of fast phase velocity and the gain of the reflex (Fig. $3 K, L$, solid blue lines). 
A Short-term ATD section

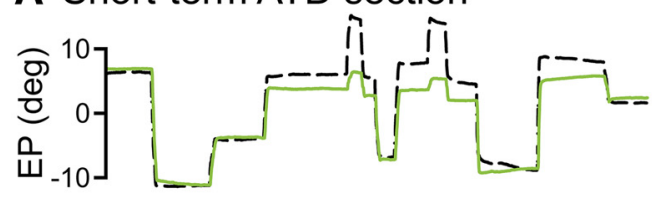

\section{Short-term MLF section}

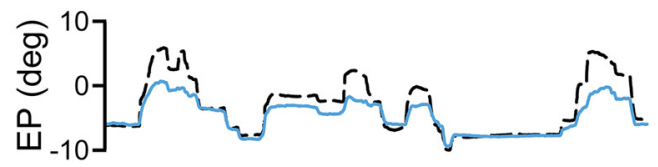

E

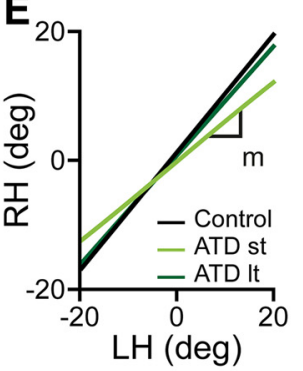

$\mathbf{F}$

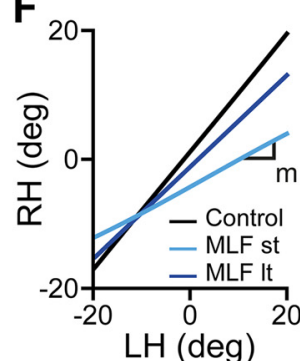

B Long-term ATD section

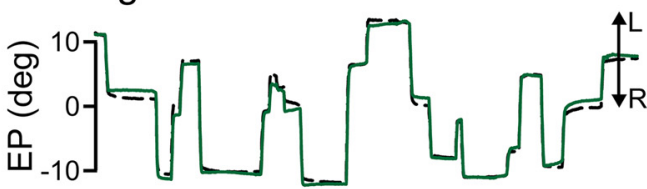

D Long-term MLF section

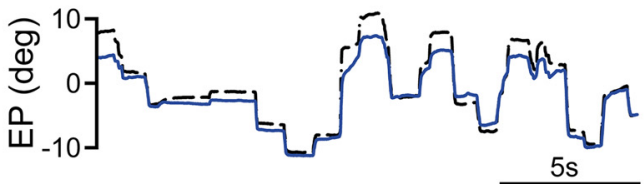

I Short-term ATD section

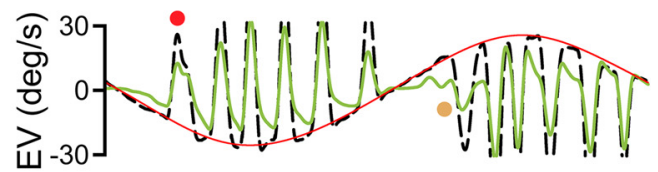
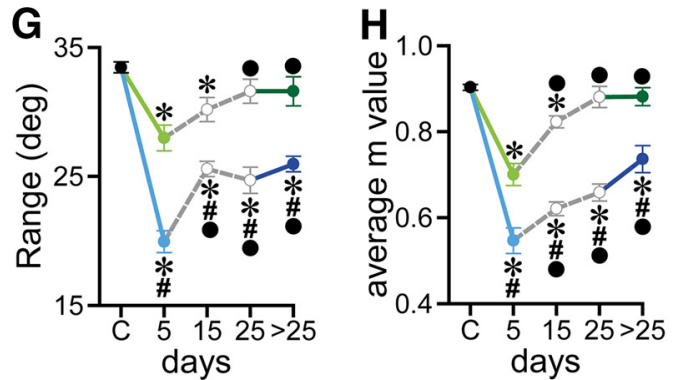

$\mathbf{J}$ Long-term ATD section

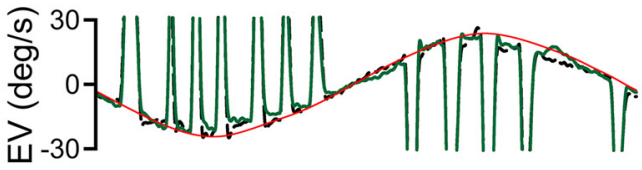

K Short-term MLF section

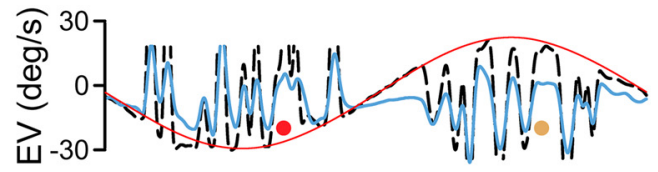

L Long-term MLF section
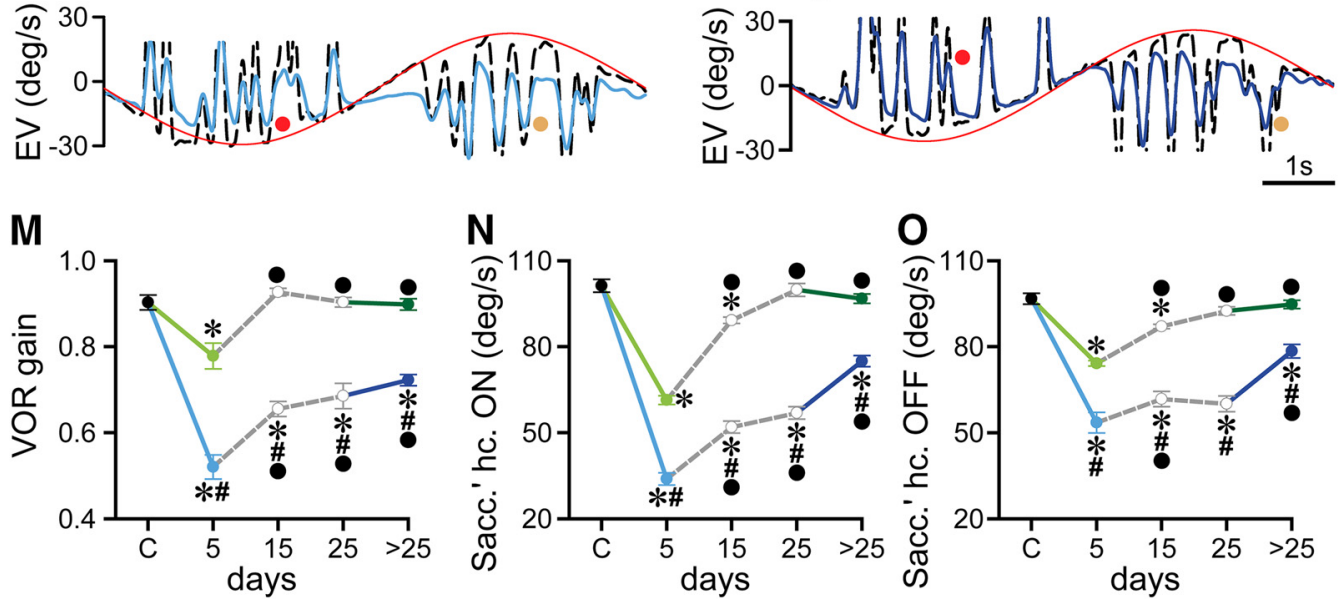

Figure 3. Changes in eye movements in response to MLF or ATD sectioning. $A-D$, Spontaneous eye movements $2 \mathrm{~d}(\boldsymbol{A}, \boldsymbol{C})$ or $26 \mathrm{~d}(\boldsymbol{B}, \boldsymbol{D})$ after ATD $(\boldsymbol{A}, \boldsymbol{B})$ or MLF $(\boldsymbol{C}, \boldsymbol{D})$ lesion. Solid lines correspond to the affected eye, while dashed lines illustrate the control eye. Upward deflections of $E P$ (in degrees) indicate eye movements to the left $(L ; R$, to the right). $E, F$, Linear regression lines between horizontal movement of both eyes, in either ATD-lesioned $(\boldsymbol{E})$ or MLF-lesioned $(\boldsymbol{F})$ animals. The slope $m$ was used as an index of conjugacy. $\mathbf{G}, \boldsymbol{H}$, Time course of the range of eye movements $(\boldsymbol{G})$ or the slope $m(\boldsymbol{H})$ after selective deafferentation ( $n \geq 21$ and $\leq 135$ intervals of 100 s of spontaneous eye movements). $\boldsymbol{I}-\boldsymbol{L}$, Vestibularly induced EV (in degrees/s) $1 \mathrm{~d}(\boldsymbol{I}, \boldsymbol{K})$ or $26 \mathrm{~d}(\boldsymbol{J}, \boldsymbol{L})$ after ATD $(\boldsymbol{I}, \boldsymbol{J})$ or $\operatorname{MLF}(K, L)$ lesioning. Head velocity is shown inverted in red for visual comparison with EV. Recordings of the affected eye are represented by colored solid lines, and the control eye velocity is represented by black dashed lines. Note the reduction in the velocity of the fast phases of the reflex in the first $5 \mathrm{~d}$ after lesioning, which returns to normal in ATD animals but not in MLF animals. Red and orange dots point to reductions in saccades occurring during the off- or on-hemicycles of the slow phases of the vestibulo-ocular reflex, respectively. $M$, Changes in the gain of the vestibulo-ocular reflex (VOR) with time, expressed as the ratio between eye and head velocity ( $n \geq 20$ and $\leq 51$ intervals of $100 \mathrm{~s}$ of VOR analyzed). $\mathbf{N}, \mathbf{0}$, Two-way ANOVA comparisons of the effect of MLF or ATD sections at different time points (short term, $5 \mathrm{~d}$ postlesion; long term, $>25 \mathrm{~d}$ postlesion) on the peak velocity of saccades (Sacc.) occurring during the on hemicycles (hc.) of the slow phases of the $\operatorname{VOR}(\boldsymbol{N})$ or during the off slow phases ( $\mathbf{0}$; Sacc.' hc. OFF; $n \geq 42$ and $n \leq 339$ VOR hemicycles analyzed for saccades). For $\boldsymbol{M - 0}$, blue and green lines correspond to data from MLF and ATD animals, respectively. Control, Black dot; short-term recordings, light-colored lines; long-term recordings, dark-colored lines. *Indicates differences with controls. Ondicates differences with respect to short-term values within the same lesion type. \#Indicates differences between the two lesions at the same time point. Data are shown as the mean \pm SEM. Two-way ANOVA, Holm-Sidak test, $p<0.05$. 
Statistical analysis confirmed that partial input depletion induced a reduction in the gain of the reflex during the first $5 \mathrm{~d}$ after lesioning that was even lower in MLF animals (control animals: $0.90 \pm 0.02, n=47$; ATD animals: $0.78 \pm 0.03, n=27$; MLF animals: $0.52 \pm 0.03, n=20$; $p<0.001$; Fig. $3 M$ ). ATD animals recovered normal values at longer time periods $(0.90 \pm 0.01$ at long term; $n=28$ ), but in the case of MLF animals gain values improved with time but never reached those described during control eye movements (for the long term, the gain was $0.72 \pm$ $0.01, n=26, p<0.001$; two-way ANOVA test, $F_{(2,3)}=6.93 ; p<$ 0.001, Holm-Sidak method for multiple comparisons; Fig. $3 M)$.

Control mean peak eye velocity of on-directed saccades was $101.2 \pm 2.2 \%$ s $(n=106)$. During vestibularly induced eye movements, we will refer to on-saccades to those occurring during the on hemicycles of the slow phases, and off-saccades to those during the off-hemicycles of the reflex (Fig. $3 \mathrm{~N}, \mathrm{O}$ ). Partial deafferentation induced a reduction in the velocity of on-saccades in the short term that was statistically lower in MLF animals (33.8 \pm $2.1 \%, n=50)$ than in ATD animals $\left(61.3 \pm 1.5^{\circ} / \mathrm{s}, n=245 ; p<\right.$ 0.001 ; Fig. $3 N$ ). In the case of ATD-sectioned animals, this value returned to normality from $15 \mathrm{~d}$ postlesion onward and remained similar to that of controls until the end of the experiments $\left(99.8 \pm 2.2^{\circ} / \mathrm{s}, n=127\right.$, for the interval $\left.15-25 \mathrm{~d}\right)$. In the case of MLF-sectioned animals, this recovery was not complete, and the mean amplitude in the long term $(75.0 \pm 2.0 \% \mathrm{~s}, n=60)$ resulted in higher values than those obtained between 0 and $5 \mathrm{~d}$ postsurgery, but lower $(p<0.001)$ than those from control and ATD long-term data (two-way ANOVA test, $F_{(2,3)}=27.29 ; p<$ 0.001 , Holm-Sidak method for multiple comparisons; Fig. $3 N$ ). Data obtained during off-directed saccades offered similar results: in the short term, there was a reduction $(p<0.001)$ in the amplitude of saccades that was more drastic for MLF animals when compared with ATD animals (control animals: $96.7 \pm$ $1.9 \%$ s, $n=106$; ATD animals: $74.2 \pm 0.9 \%, n=245$; MLF animals: $53.5 \pm 3.5^{\circ}$ s, $n=50$; Fig. 30 ). The effects of partial deafferentation were transitory, and long-term data were different $(p<0.001)$ from short-term data between the two types of lesion (ATD animals: $94.7 \pm 1.4 \%, n=124$; MLF animals: $78.4 \pm 2.3 \%, n=60$ ), although only ATD animals recovered the normal amplitude of off-directed saccades (two-way ANOVA test, $F_{(2,3)}=13.44 ; p<0.001$, Holm-Sidak method for multiple comparisons; Fig. 3O).

\section{Firing pattern of partially deafferented medial rectus motoneurons during spontaneous eye movements}

Medial rectus motoneurons exhibited a tonic-phasic firing pattern, which resembled that described for abducens motoneurons, as previously reported (de la Cruz et al., 1989). Thus, the firing rate increased tonically with successive ipsilateral eye fixations toward more adducting positions. During adducting eye movements, medial rectus motoneurons fired bursts of action potentials whose amplitude was directly proportional to the velocity of the eye (Fig. $4 A$, asterisk), while abducting movements were related to decreases in firing rate (Fig. $4 A$, dots). The slope of the regression line obtained after plotting firing rate versus eye position indicated the neuronal sensitivity to eye position, $k_{s}$ (Fig. $4 E$, black line), and that obtained after plotting the firing rate versus eye velocity was $r_{s}$, which represented the neuronal sensitivity to eye velocity (Fig. $4 G$, black line).

After both MLF and ATD lesioning, the overall firing rate was reduced, bursts of spikes during on-directed saccades were less pronounced (Fig. 4B,C, asterisks), and off-directed saccades were associated with smaller decreases in neuronal firing, that, however, often implicated complete firing pauses (Fig. $4 B, C$, dots). Nevertheless, long-term experiments did not show any firing alteration in ATD-lesioned animals, with normal firing rates and a correct modulation of activity in relation to ondirected saccades (Fig. $4 D$, asterisks) and off-directed saccades (Fig. 4D, dots). This recovery was absent in MLF-lesioned animals throughout the entire span of the experiments (Fig. $4 B$ ).

Plots representing firing rate versus eye position (Fig. $4 E$ ) or velocity (Fig. 4G) showed higher slopes, and thus higher $k_{s}$ and $r_{s}$ values, in control (black lines) and long-term ATD recordings (dark green line) when compared with short-term recordings (ATD, light green and MLF, light blue) and long-term MLF data (dark blue).

The mean control $k_{s}$ value was $4.4 \pm 0.1 \mathrm{sp} / \mathrm{s} /{ }^{\circ}$ ( $\mathrm{sp}=$ spikes; $n=98$; Fig. $4 F)$. Partial deafferentation produced a reduction $(p<0.001)$ in the $k_{s}$ values for both types of lesions in the short term (ATD animals: $2.8 \pm 0.2 . n=34$; MLF animals: $1.6 \pm 0.2$ $\left.\mathrm{sp} / \mathrm{s} /{ }^{\circ}, n=13\right)$, higher in the case of MLF animals $(p=0.007)$. There was a rapid recovery in $k_{s}$ values in the case of ATDdeprived motoneurons (Fig. $4 F$ ). Thus, $15 \mathrm{~d}$ after ATD lesioning, the $k_{s}$ value was $3.8 \pm 0.2 \mathrm{sp} / \mathrm{s} /{ }^{\circ}(n=33)$ and remained unchanged in the long term $\left(4.2 \pm 0.3 \mathrm{sp} / \mathrm{s} /{ }^{\circ}, n=23\right.$, for data recorded $>25 \mathrm{~d}$ after lesion). On the contrary, $k_{s}$ values of MLF animals did not recover with time and were lower than data for controls and ATD animals for the same time point in any time selected $\left(2.8 \pm 0.2 \mathrm{sp} / \mathrm{s}^{\circ}{ }^{\circ} ; n=21, p<0.001,>25 \mathrm{~d}\right.$ after lesion; two-way ANOVA test, $F_{(2,3)}=2.75 ; p=0.012$, Holm-Sidak method for multiple comparisons; Fig. $4 F$ ). Similar results were obtained for the analysis of $r_{s}$ (Fig. $4 H$ ): the mean control value $\left(0.48 \pm 0.02 \mathrm{sp} / \mathrm{s}^{\circ}, n=98\right)$ was reduced after both types of lesions in the short term (ATD group: $0.28 \pm 0.02 \mathrm{sp} / \mathrm{s} /{ }^{\circ}, n=34$; MLF group: $\left.0.15 \pm 0.02 \mathrm{sp} / \mathrm{s} /{ }^{\circ}, n=13 ; p<0.001\right)$. In the case of MLF animals, this value remained lower $(p<0.001)$ than that for controls for the whole experimental period $\left(0.21 \pm 0.02 \mathrm{sp} / \mathrm{s} /{ }^{\circ}\right.$, $n=21,>25 \mathrm{~d}$ after lesioning). On the contrary, vestibular input depletion only produced short-term effects, which returned to normality from $15 \mathrm{~d}$ after lesioning onward (15-25 d interval: $0.42 \pm 0.03 \mathrm{sp} / \mathrm{s} /{ }^{\circ}, n=22$; two-way ANOVA test, $F_{(2,3)}=3.10$; $p=0.005$, Holm-Sidak method for multiple comparisons; Fig. $4 H)$.

Therefore, $k_{s}$ and $r_{s}$ values in medial rectus motoneurons decreased in the first $5 \mathrm{~d}$ after ATD or MLF lesioning, but recovered with time only after ATD sectioning.

\section{Synaptic input depletion impairs eye-related firing rate during the vestibulo-ocular reflex}

Figure $5 A$ shows eye position and velocity, as well as related motoneuron firing rate, during the performance of the vestibuloocular reflex for a control cell. Eye velocity nearly overlaps with head velocity, so that the control gain was close to 1 . Control motoneuron discharge closely resembled eye position, as seen during the slow phases of the reflex, with bursts or pauses of spikes during the on- or off-directed fast phases of the nystagmus, respectively. The modulation of motoneuron firing rate also changed after selective deafferentation during vestibularly induced eye movements. In the short term, both ATD and MLF sections had similar effects on firing discharge: a reduction in the modulation related to both eye position and velocity (Fig. 5C). In contrast, long-term recordings showed differences between these two groups. Thus, MLF animals produced similar results when compared with those obtained in the first days after lesioning (Fig. 5B). However, cells recorded from $25 \mathrm{~d}$ onward after ATD transection modulated their firing rate in relation to eye move- 


\section{A Control}
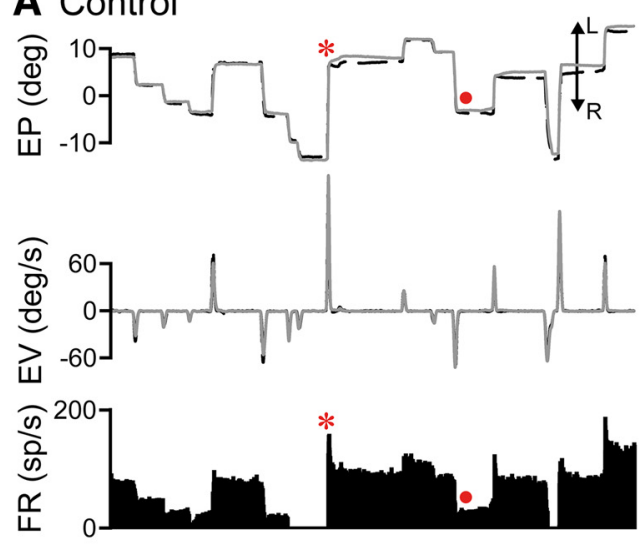

C Short-term ATD section

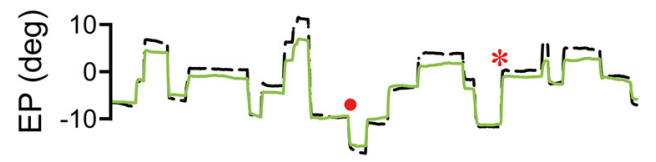
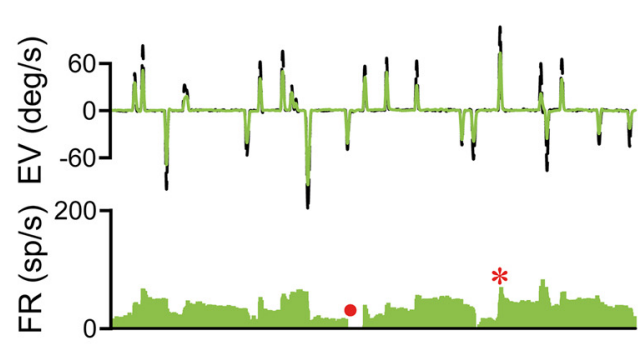

E

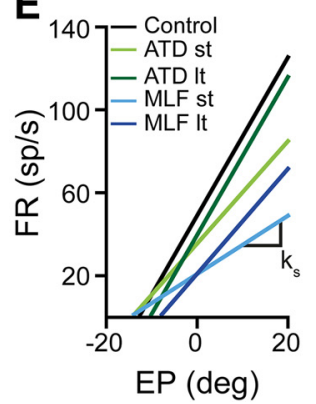

$\mathbf{F}$

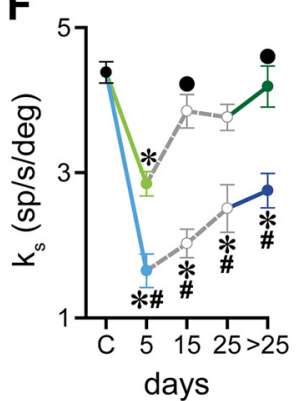

B Long-term MLF section
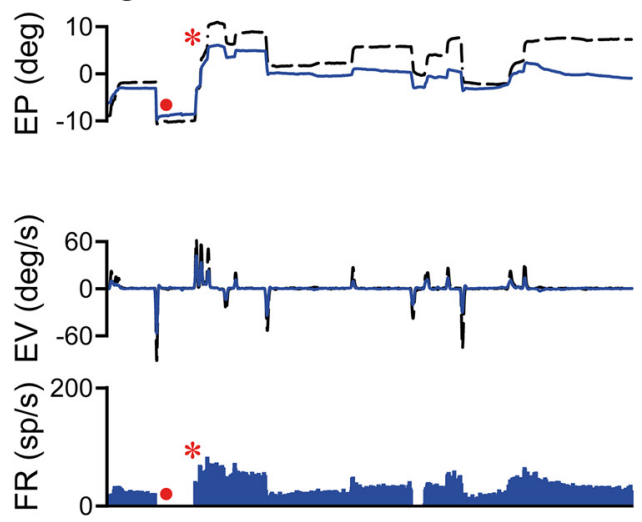

D Long-term ATD section
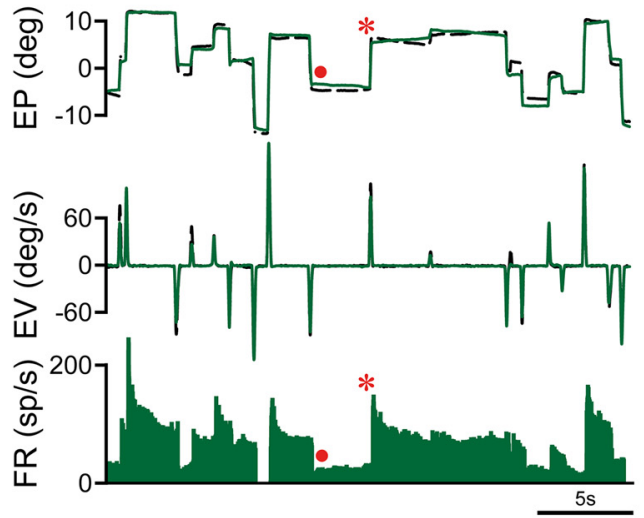

G

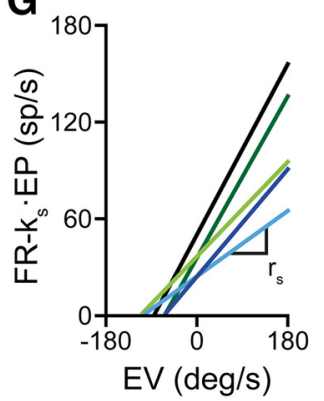

H

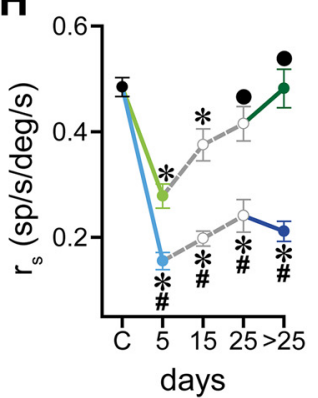

Figure 4. Effects of deafferentation on medial rectus motoneuron firing pattern during spontaneous eye movements. $A-D$, From top to bottom, horizontal right EP and EV and instantaneous FR (in spikes/s) in a control animal (A), $30 \mathrm{~d}$ after MLFsection (B), and either $2 \mathrm{~d}(\boldsymbol{C})$ or $39 \mathrm{~d}(\boldsymbol{D})$ after ATD transection. Land R indicate movements toward the left and right, respectively. Asterisks indicate the occurrence of on-directed saccades and the associated bursts in neuronal firing (note the reduced bursts in $\boldsymbol{B}$ and $\boldsymbol{C}$ but not in $\boldsymbol{D}$ ). Dots point to off-directed saccades, related to decreases in firing rate. $\boldsymbol{E}-\boldsymbol{H}$, Correlation between firing rate and either horizontal $\mathrm{EP}(\boldsymbol{E})$ or $\mathrm{EV}(\boldsymbol{G})$. The slope of the linear regression lines is the neuronal sensitivity to eye position $\left(\boldsymbol{k}_{s}\right.$ in $\left.\boldsymbol{E}\right)$ or velocity $\left(r_{s}\right.$ in $\left.\boldsymbol{G}\right)$. Note an st decrease in $k_{s}$ and $r_{s}$ values after both types of lesion, which in the case of ATD animals returns to control in the lt. Mean and SEM for $k_{s}$ and $r_{s}$ values were calculated and compared ( $\boldsymbol{F}$ and $\boldsymbol{H}$, respectively). Color codes are indicated in the figure. For $\boldsymbol{F}, \boldsymbol{H}_{\text {, }}{ }^{*}$ indicates differences with controls, $\bigcirc$ indicates differences with respect to short-term values, and\# indicates differences between lesion groups at the same time point. Two-way ANOVA, Holm-Sidak test, $p<0.05$. The number of cells analyzed at each time point was 98 control cells, 34, 33, 22, and 23 for st, between 6 and $15 \mathrm{~d}$, between 16 and $25 \mathrm{~d}$, and It in ATD animals, respectively, and 13,31, 18, and 21 cells for MLF animals at the same time points, respectively. The same cells are analyzed for $\boldsymbol{F}, \boldsymbol{H}$.

ments in a way that resembled that observed in the control situation (Fig. 5D). Plots of firing rate versus eye position or velocity were made to obtain the slopes of the regression lines, which are named neuronal sensitivity to eye position or to eye velocity during the vestibulo-ocular reflex (Fig. $5 E, I, k_{v}$ or $r_{v}$, respectively). As expected, statistical analyses (two-way ANOVA test: $k_{v}: F_{(2,3)}=$ 2.32, $p=0.033 ; r_{v}: F_{(2,3)}=2.72, p=0.014$; Holm-Sidak method for multiple comparisons) showed a decrease in both $k_{v}$ and $r_{v}$ values in the first $5 \mathrm{~d}$ after lesioning [Fig. $5 F$, J, respectively; $k_{v}$ values: control animals, $5.1 \pm 0.3 \mathrm{sp} / \mathrm{s} /{ }^{\circ}$; ATD animals, $2.7 \pm 0.2 \mathrm{sp} / \mathrm{s} /{ }^{\circ}$; MLF animals, $1.6 \pm 0.3 \mathrm{sp} / \mathrm{s} /{ }^{\circ} ; r_{v}$ values: control animals, $0.72 \pm 0.03$ $\mathrm{sp} / \mathrm{s}^{\circ}$; ATD animals, $0.43 \pm 0.03 \mathrm{sp} / \mathrm{s}^{\circ}{ }^{\circ}$; MLF animals, $0.39 \pm$
$0.07 \mathrm{sp} / \mathrm{s} /{ }^{\circ}$ (control animals, $n=56$; ATD animals, $n=23 ;$ MLF animals, $n=11) ; p<0.001]$. This decrease remained throughout the time course of the experiments in the case of MLFsectioned animals for both $k_{v}$ and $r_{v}$ (Fig. $5 F, J$, respectively). However, the deprivation of vestibular input did not produce permanent changes, and data from $15 \mathrm{~d}$ postlesion onward were similar to those for control animals (Fig. $5 F, J$ ).

To perform a more accurate analysis and reach a better comprehension of the role of abducens and vestibular inputs on medial rectus motoneurons, we analyzed separately neuronal sensitivity during on-directed and off-directed slow phases of the reflex. Again, the reduction in $k_{v}$ and $r_{v}$ values observed a few days 

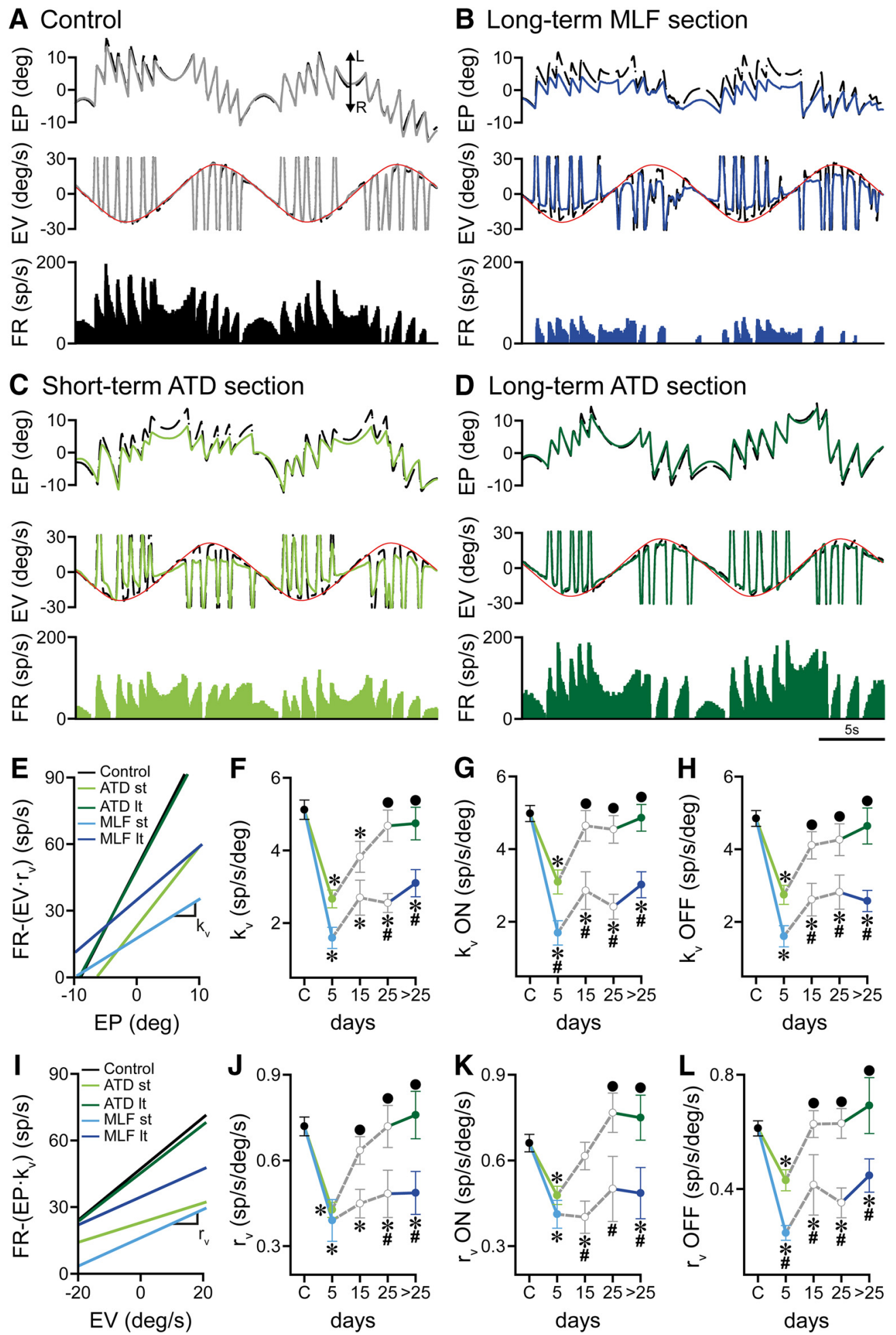

Figure 5. Changes in firing pattern during vestibularly induced eye movements. $\boldsymbol{A}-\boldsymbol{D}$, From top to bottom: horizontal $E P$ and $E V$, and $F R$ in a control animal $(\boldsymbol{A}), 30 \mathrm{~d}$ after $M L F$ transection $(\boldsymbol{B})$, and 3 or $44 \mathrm{~d}$ after ATD lesioning $\left(\boldsymbol{C}\right.$ and $\boldsymbol{D}$, respectively). Inverted head velocity is indicated in middle plots by a red line. $\boldsymbol{E}_{\boldsymbol{\prime}} \boldsymbol{I}$, Neuronal sensitivities to eye position $\left(k_{v^{\prime \prime}} \boldsymbol{E}\right)$ and velocity $\left(\boldsymbol{r}_{v^{\prime \prime}} \boldsymbol{I}\right)$ calculated as the slope of the regression line obtained after plotting FR vs EP or EV, respectively. $\boldsymbol{F}-\boldsymbol{H}$, Time course of $k_{v}$ values $(\boldsymbol{F})$, and $k_{v}$ values during on-directed $(\boldsymbol{G})$, or off-directed slow phases of the VOR $(\boldsymbol{H})$. $\boldsymbol{J}-\boldsymbol{L}$, Time course of $r_{v}$ values $(\boldsymbol{J})$, and $r_{v}$ values during on-directed $(\boldsymbol{K})$ or off-directed slow phases of the reflex $(\boldsymbol{L})$. Color codes are indicated in the figure. For $\boldsymbol{F}-\boldsymbol{H}$ and $\boldsymbol{J}-\boldsymbol{L}$, ${ }^{*}$ indicates differences with controls. indicates differences with short-term data within the same lesion type, and \# indicates differences between lesion types at the same time point. Data are shown as the mean $\pm \mathrm{SEM}$. Two-way ANOVA, Holm-Sidak test, $p<0.05$; the number of cells was 56 in the control situation, 23, 24, 19, and 20 in ATD animals, and 11, 12, 11, and 13 cells in MLF animals at st, between 6 and 15 , between 16 and $25 \mathrm{~d}$, and It, respectively. Same cells analyzed in $\boldsymbol{F}-\boldsymbol{H}$ and $\boldsymbol{J} \boldsymbol{-} \boldsymbol{L}$.

after lesioning during on-directed (Fig. $5 G, k_{v} \mathrm{ON}$; Fig. $5 K, r_{v} \mathrm{ON}$; two-way ANOVA: $k_{v} \mathrm{ON}, F_{(2,3)}=2.16, p=0.046 ; r_{v} \mathrm{ON}, F_{(2,3)}=$ 2.39, $p=0.028$; Holm-Sidak method for multiple comparisons) and off-directed slow phases (Fig. $5 H, k_{v}$ OFF; Fig. $5 L, r_{v}$ OFF; two-way ANOVA: $k_{v} \mathrm{ON}, F_{(2,3)}=2.22, p=0.041 ; r_{v} \mathrm{ON}, F_{(2,3)}=$ 2.36, $p=0.030$; Holm-Sidak method for multiple comparisons) was permanent after abducens input depletion, but returned to normal values in ATD animals. 

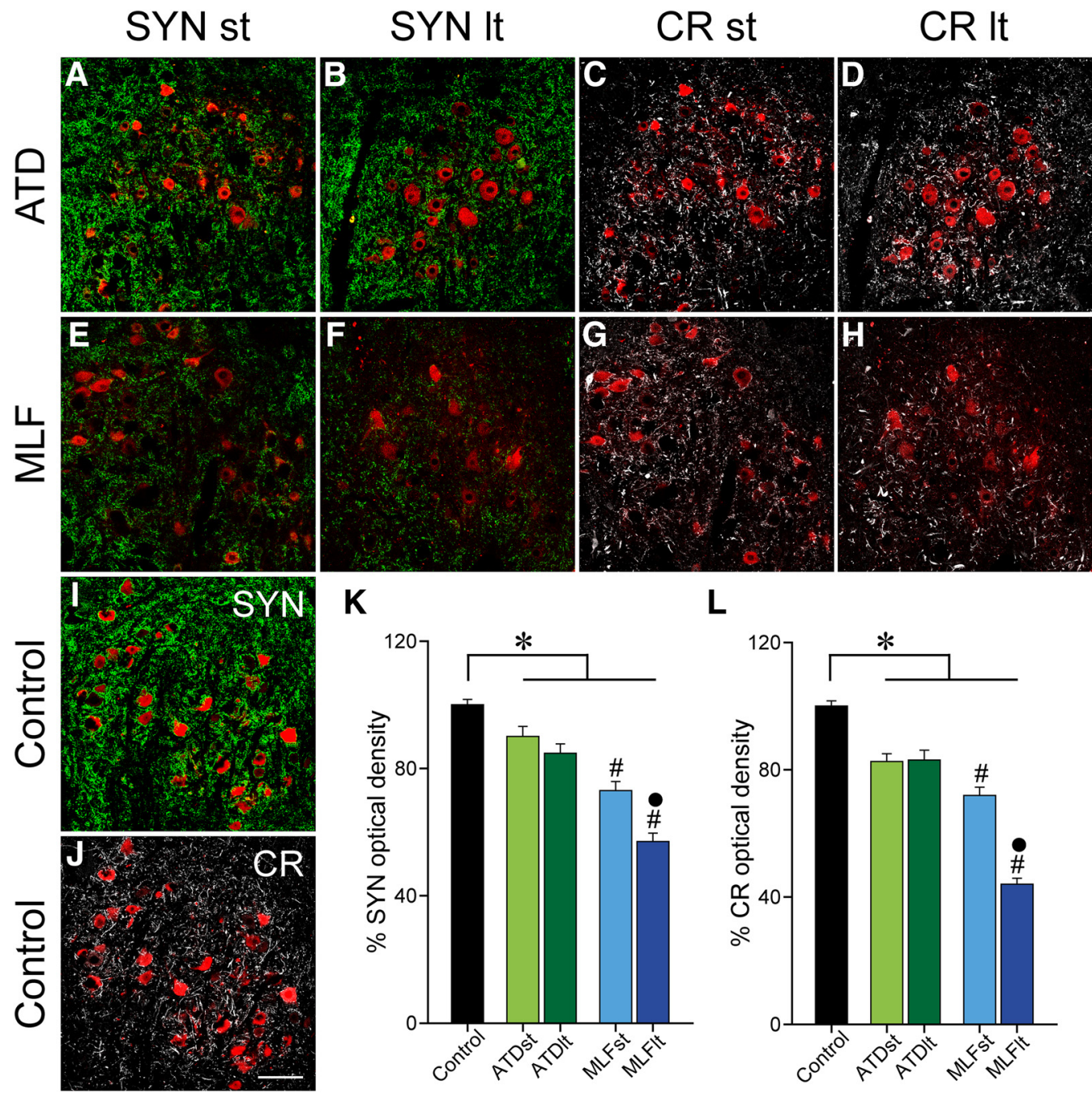

$\mathbf{L}$

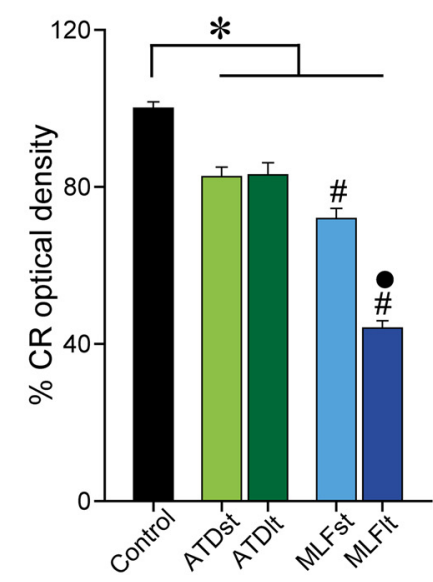

Figure 6. Synaptic coverage in the neuropil measured as optical density. $A-H$, Confocal images of the medial rectus motoneuron subdivision, identified by retrograde rhodamine staining, in red, illustrating the presence of synaptophysin-positive terminals $(\boldsymbol{A}, \boldsymbol{B}, \boldsymbol{E}, \boldsymbol{F} ; \mathrm{SYN}$, in green) and calretinin-positive axons $(\boldsymbol{C}, \boldsymbol{D}, \boldsymbol{G}, \boldsymbol{H} ; \boldsymbol{C R}$, pseudocolored in white) in ATD $(\boldsymbol{A}-\boldsymbol{D})$ and $M L F(\boldsymbol{E}-\boldsymbol{H})$ animals. $\boldsymbol{I}, J$, Images obtained from the control side. $\boldsymbol{K}, \boldsymbol{L}$, Comparisons of optical density (normalized to control values) at the neuropil of the nucleus in sections stained against synaptophysin $(\boldsymbol{K})$ or calretinin $(\boldsymbol{L})$. For $\boldsymbol{K}, \boldsymbol{L},{ }^{*}$ indicates differences between the marked groups, $\boldsymbol{O}$ indicates differences with short-term data within the same lesion type, and \# indicates differences between lesion types at the same time point; $n \geq 210$ and $\leq 1300$ measurements of optical density (same for $\boldsymbol{K}, \boldsymbol{L}$ in the double immunofluorescence). Two-way ANOVA, Holm-Sidak test, $p<0.05$. Scale bar, $J$ (for $\boldsymbol{A}-\boldsymbol{J}), 100 \mu \mathrm{m}$.

Reduction in synaptic coverage of medial rectus motoneurons after partial deafferentation

Partial deafferentation produced a reduction in both synaptophysin (Fig. 6A, B,E,F) and calretinin (Fig. 6C,D, G,H) staining in the neuropil around medial rectus motoneurons, measured as the percentage of optical density in relation to the control side (Fig. 6I,J). Statistical analysis revealed that this reduction was more drastic after MLF sectioning when compared with ATD data at any time point (Fig. $6 K, L$ ) in accordance with previous reports pointing to a segregated innervation of medial rectus motoneurons (Nguyen et al., 1999). No differences were found between short-term and long-term data in ATD animals. However, MLF transection produced a decrease in both synaptophysin and calretinin staining that increased with time. Nevertheless, neither synaptophysin nor calretinin immunostaining in the neuropil yielded the control level in the long term after both types of lesioning, although the reduction in MLF-sectioned animals was higher than in ATD-sectioned animals (Fig. $6 K, L$ ). Thus, in the long term, synaptophysin optical density in the neuropil was $57.0 \pm 2.7 \%(n=380$ measurements $)$ after MLF transection, which was significantly lower $(p<0.01)$ than after ATD lesion- ing $(84.8 \pm 3.0 \% ; n=380)$, and in both cases synaptophysin optical density was lower than in control animals $(100 \pm 1.7 \%$; $n=1300 ; p<0.01$, two-way ANOVA, $F_{(1,2)}=4.35 ; p=0.013$, Holm-Sidak method for multiple comparisons).

MLF and ATD transection also produced a decrease in the synaptic coverage around cell bodies, measured as the percentage of the soma perimeter covered by synaptophysin- or calretininpositive terminals, as shown in Figure 7A-E (synaptophysin) and Figure $7 F-J$ (calretinin). Neither in the short term nor in the long term did we find changes in soma diameter or perimeter due to different lesions (two-way ANOVA: soma diameter, $F_{(1,2)}=0.25$, $p=0.779$; soma perimeter, $F_{(1,2)}=1.179, p=0.308$; HolmSidak method for multiple comparisons; not illustrated). Thus, control motoneurons showed a large percentage of their soma surrounded by bouton-like processes stained against synaptophysin (Fig. $7 K ; 38.2 \pm 0.5 \%, n=268$ cells). Calretinin staining allowed the specific labeling of abducens and vestibular innervation, which covered $\sim 29.0 \pm 0.5 \%$ of the soma perimeter (Fig. $7 L ; n=268)$. On the contrary, these terminal processes were scarcer after partial deafferentation at any time point and for both types of lesions. Thus, the percentage of the soma perimeter cov- 

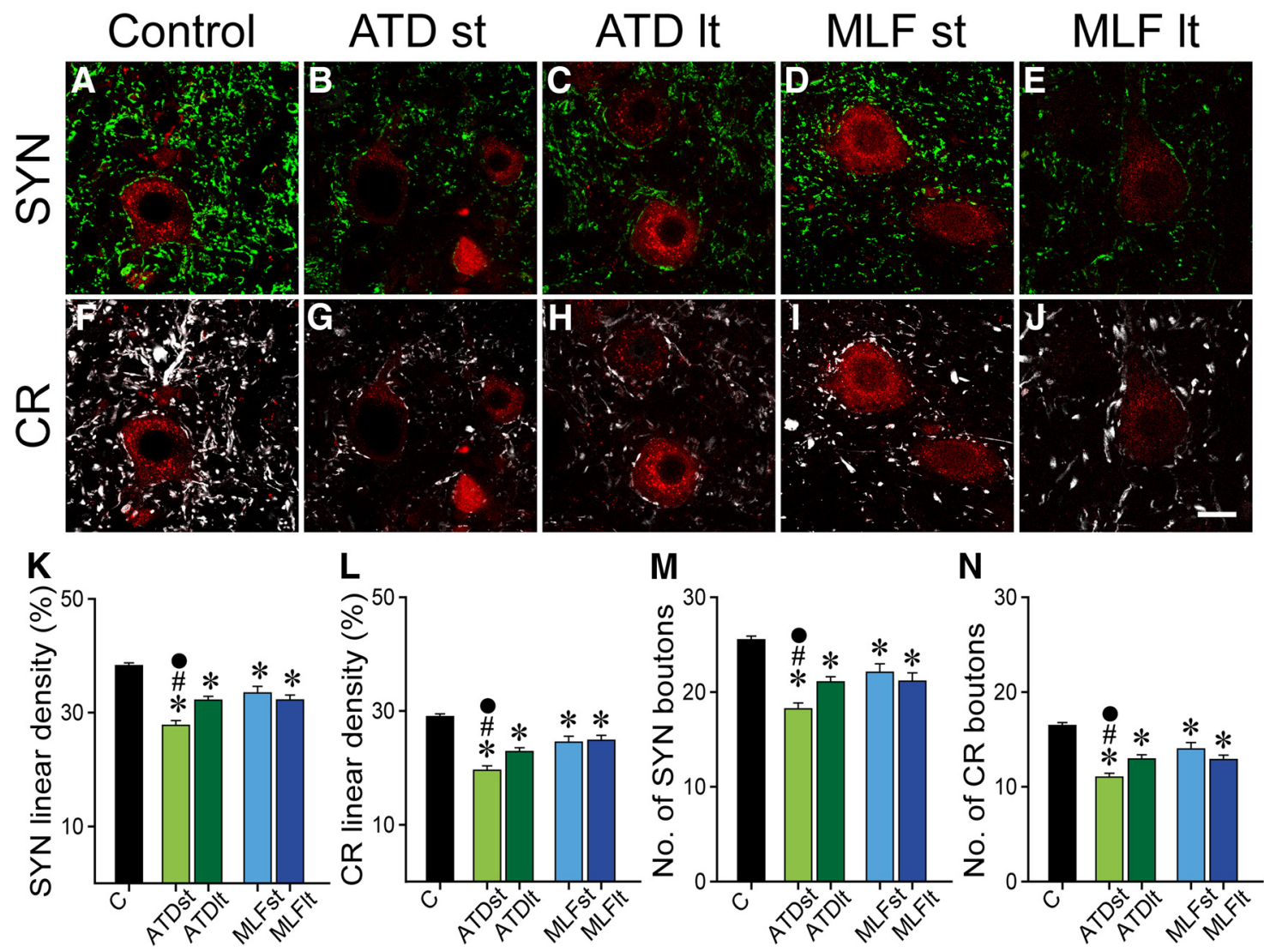

Figure 7. Synaptic coverage of medial rectus motoneuron somata after selective partial deafferentation. $\boldsymbol{A}-\boldsymbol{E}$, Confocal microscope images of medial rectus motoneurons (labeled with rhodamine, in red) and synaptophysin-immunoreactive terminals (SYN, in green) surrounding neuron cell bodies in control ( $\boldsymbol{A})$, short-term ATD (B), long-term ATD (C), short-term MLF (D), and long-term MLF $(\boldsymbol{E})$ animals. $\boldsymbol{F}-\boldsymbol{J}$, Same as above but for calretinin-immunoreactive terminals (CR, pseudocolored in white). $\boldsymbol{K}, \boldsymbol{M}$, Bar charts comparing mean \pm SEM values of the synaptic coverage around soma perimeter $(\boldsymbol{K})$ and number of boutons $(\boldsymbol{M})$ in control (blackbar), short-term ATD (light green), long-term ATD (dark green), short-term MLF (light blue), and long-term MLF (darkblue) groups using immunostaining against synaptophysin(SYN). $L, \boldsymbol{N}$, Sameas $\boldsymbol{K}, \boldsymbol{M}$, but forterminals stained against CR. For $\boldsymbol{K}-\boldsymbol{N},{ }^{*}, \#$, and indicate differences with controls, between lesions at the same time point, or between time points in the same lesion group, respectively. Two-way ANOVA, Holm-Sidak test, $p<0.05 ; n \geq 32$ and $\leq 268$ cells. Same cells analyzed for $\boldsymbol{K}-\boldsymbol{N}$. Scale bar, $\boldsymbol{J}$ (for $\boldsymbol{A}-\boldsymbol{J}$ ), $25 \mu \mathrm{m}$.

ered by synaptophysin-immunopositive terminals was reduced after lesioning (short-term ATD: $27.8 \pm 0.8 \% ; n=96 ; p<0.001$, vs controls; short-term MLF: $33.4 \pm 1.2 \% ; n=32 ; p=0.001$, vs controls) and did not recover with time (long-term ATD: $32.2 \pm$ $0.7 \% ; n=101 ; p<0.001$, vs controls; long-term MLF: $32.2 \pm$ $0.9 \% ; n=71 ; p<0.001$, vs controls; Fig. $7 K)$. Statistical analysis revealed that the higher reduction with respect to controls $(p<$ 0.001 ) was observed $3 \mathrm{~d}$ after ATD transection (Fig. $7 K$, ATD st), but this value increased 2 months after lesioning (Fig. 7K, ATD lt; $p<0.001$ ), suggesting a synaptic plastic process. MLF transection produced the less drastic reduction, which, however, did not improve with time (Fig. $7 \mathrm{~K}$; two-way ANOVA test, $F_{(1,2)}=6.34 ; p=$ 0.002 , Holm-Sidak method for multiple comparisons). These results are in agreement with previous research indicating a stronger vestibular innervation at the somatic level of medial rectus motoneurons when compared with abducens inputs (Nguyen et al., 1999). To assess whether changes in synaptic coverage were due to differences in the number of boutons contacting medial rectus motoneurons, we also counted and compared the number of synaptophysin-positive boutons around the soma perimeter (Fig. 7M). Results were consistent with synaptic coverage data: ATD transection produced a more drastic reduction in the number of somatic boutons (18.2 $\pm 0.6, n=96$, short term), which was lower $(p<0.001)$ than that for controls $(25.5 \pm$ $0.4, n=268$ ) but was also lower than long-term ATD and shortterm MLF data (long-term ATD: $21.0 \pm 0.6, n=101, p=0.002$; short-term MLF: $22.1 \pm 0.9, n=32, p=0.003$; two-way ANOVA, $F_{(1,2)}=4.18 ; p=0.016$, Holm-Sidak method for multiple comparisons). Interestingly, according to the present data, synaptic coverage in long-term ATD animals increased in relation to the short-term data, although it was still lower than in controls (Fig. $7 \mathrm{~K}, \mathrm{M}$ ). This result implies that after ATD transection there must be a rapid degeneration of terminals around the soma (ATD st), but that 2 months after lesioning (ATD lt) other new terminals covered the gaps left by the degenerated boutons, likely by axonal sprouting of intact MLF axons, which could explain the recovery in the motor function.

To determine the changes in vestibular innervation after MLF transection, or abducens innervation after ATD lesion, we also stained axon terminals against calretinin. Results, both for synaptic coverage and number of boutons around cell bodies, were similar to those described for synaptophysin-stained terminals (Fig. $7 L, N$; two-way ANOVA: synaptic coverage, $F_{(1,2)}=3.21$, $p=0.041$; number of boutons, $F_{(1,2)}=3.68 ; p=0.026$; HolmSidak method for multiple comparisons).

Induction of GAP-43 expression in medial rectus motoneuron inputs after partial deafferentation

To ascertain the possible molecular bases for this sprouting, we performed immunostaining against GAP-43, a protein that has been associated with axon growth. 
Images of short-term animals showed an intense GAP-43 labeling after both ATD (Fig. 8A) and MLF (Fig. 8C) transections. However, images obtained in the long term (Fig. 8B, ATD animals, D, MLF animals), showed a scarce staining, similar to that observed on the control side (Fig. 8E). Comparisons of optical density in the neuropil, normalized to the control side of the same section, confirmed the following qualitative observations: partial deafferentation produced an increased expression of GAP-43 in the remaining intact axons, which was more intense $(p<0.001)$ after ATD transection (336.1 $\pm 14.5 \%, n=258$ measurements) when compared with MLF transection $(179.2 \pm 8.0 \%, n=210)$. However, 2 months after lesioning, all values returned to normality (ATD: $95.2 \pm 4.9 \%, n=286$; MLF: $110.9 \pm 9.3 \%, n=145$; control: $100 \pm 3.3 \%, n=899$; two-way ANOVA, $F_{(1,2)}=213.124 ; p<0.001$, Holm-Sidak method for multiple comparisons; Fig. $8 F)$. These results indicated that the synaptic remodeling found 2 months after lesioning correlated well with an increase in GAP-43 expression, especially after ATD lesioning. However, in the case of MLF animals, this change was not enough to induce an increase in medial rectus motoneuron inputs.

\section{Increased neurotrophin expression in partially deafferented medial rectus motoneurons}

Immunocytochemistry against BDNF, NT-3, and NGF was performed to elucidate the possible trophic mechanisms implicated in axon sprouting after partial deafferentation. Control medial rectus motoneurons were positive for the three neurotrophins (Fig. $9 A-C)$. The three different antibodies produced a similar homogeneous labeling of motoneuronal somata.

Neurotrophins were also present in medial rectus motoneurons after selective deafferentation at any analyzed time point. However, images obtained in the short term, after both ATD (Fig. 9D-F) or MLF (Fig. 9J-L) transection, exhibited a more intense staining when compared with motoneurons present on the control side of the same section. Two months after lesioning, however, immunostaining was weak and resembled that obtained in control cells (Fig. 9G-I, ATD animals, $M-O$, MLF animals).

Analysis of cytoplasmic optical density, expressed as percentages relative to the control side, offered similar results for BDNF (Fig. 9P), NT-3 (Fig. 9Q), or NGF (Fig. 9R). In the short term, there was a statistically significant increase in BDNF (ATD, $114.6 \pm 3.0 \%$; MLF, $110.1 \pm 2.0 \% ; n \geq 22$ and $n \leq 161 ; p<$ 0.001 ), NT-3 (ATD, $121.2 \pm 4.9 \%$; MLF, $117.8 \pm 2.8 \%$; $n \geq 24$ and $n \leq 138 ; p<0.001$ ), and NGF (ATD, $118.8 \pm 4.2 \%$; MLF, $110.1 \pm 1.9 \% ; n \geq 17$ and $n \leq 169 ; p<0.001)$ with respect to controls (Fig. 9P-R; two-way ANOVA: BDNF, $F_{(1,2)}=9.69$; NT-3:
$F_{(1,2)}=7.16$; NGF: $F_{(1,2)}=6.76 ; p<0.001$, Holm-Sidak method for multiple comparisons). However, there were no differences in neurotrophin staining between long-term and control motoneurons, indicating a correlation between the increase in neurotrophin and GAP-43 labeling.

\section{Discussion}

The present study compares for the first time the role of the two main pontine inputs to medial rectus motoneurons on their discharge characteristics by lesion experiments. MLF lesions produced more drastic and long-lasting changes in both eye movements and motoneuron firing rate than ATD sections. On the other hand, long-term data pointed to a higher plastic capability of the MLF lesions than the ATD lesions. Plastic rearrangement of remaining inputs might be mediated by trophic factors through the regulation of GAP-43 expression. 

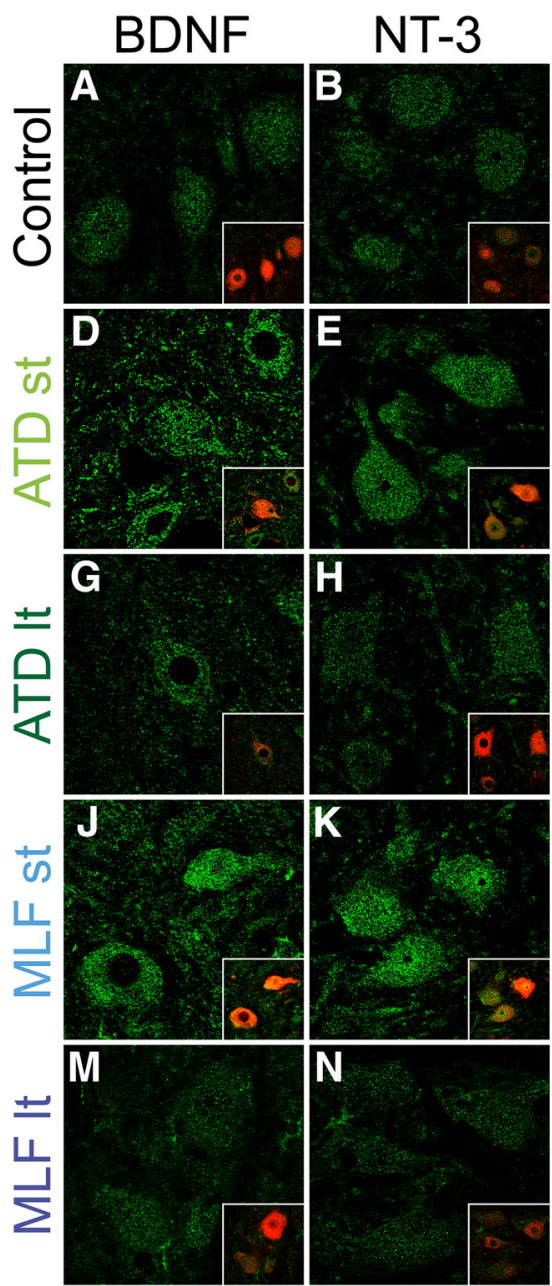
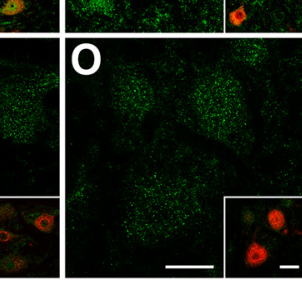
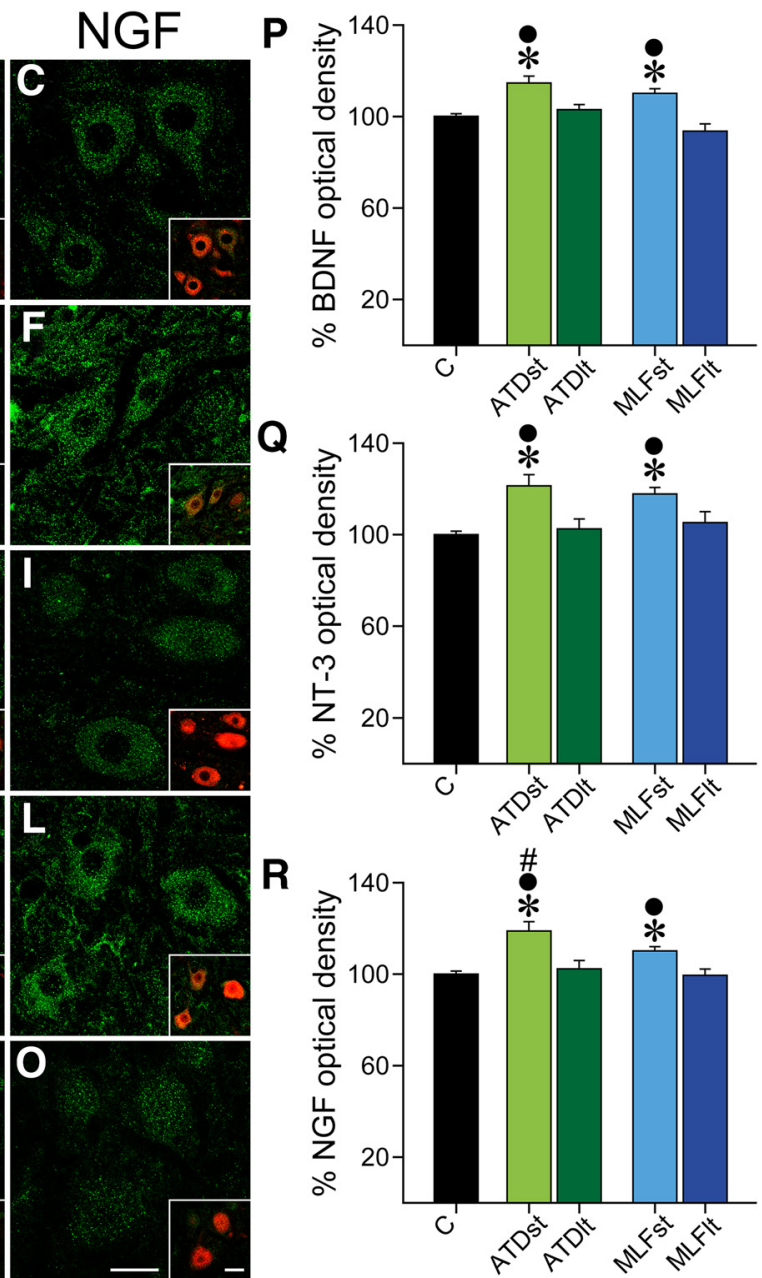

Figure 9. Changes in neurotrophin expression in response to partial deafferentation. $\boldsymbol{A}-\mathbf{0}, \mathrm{BDNF}, \mathrm{NT}-3$, and NGF immunoreactivity in control medial rectus motoneurons ( $\boldsymbol{A}-\boldsymbol{C})$, after short-term and long-term ATD transection ( $\boldsymbol{D}-\boldsymbol{F}$ and $\mathbf{G}-\boldsymbol{I}$, respectively), and after short-term and long-term MLF lesioning ( $\boldsymbol{J}-\boldsymbol{L}$ and $\boldsymbol{M}-\mathbf{0}$, respectively). Note the stronger staining in short-term images for the three neurotrophins. Motoneurons were identified by retrograde rhodamine labeling (in red, insets). $\boldsymbol{P}-\boldsymbol{R}$, Comparisons of the optical density in the cell bodies of medial rectus motoneurons among the five different groups for BDNF $(\boldsymbol{P})$, NT-3 ( $\boldsymbol{Q})$, or NGF $(\boldsymbol{R})$ staining. Control, Black bar; short-term ATD, light green; long-term ATD, dark green; short-term MLF, light blue; and long-term MLF, dark blue. For $\boldsymbol{P}-\boldsymbol{R},{ }^{*}$ indicates differences with control, \# indicates differences between lesions at the same time point, and $\mathbf{O}$ indicates differences between time points in the same lesion group. Two-way ANOVA, Holm-Sidak test, $p<0.05$. For BDNF $(\boldsymbol{P}), n \geq 22$ and $\leq 161$ cells; for NT-3 ( $\boldsymbol{Q}) n \geq 24$ and $\leq 138$ cells; and for NGF $(\boldsymbol{R}) n \geq 17$ and $\leq 169$ cells. Scale bars: $\mathbf{O}$ (for $\boldsymbol{A}-\mathbf{0}$ ), $25 \mu$ m; insets, $30 \mu \mathrm{m}$.

\section{Short-term effects of selective medial rectus motoneuron deafferentation}

Medial rectus motoneuron partial deafferentation involved a reduction in the motor range of the ipsilateral eye. This reduction was appreciated in ATD animals, but changes were more drastic after MLF sectioning. As previously described (de la Cruz et al., 2000), cats with a transection of the MLF were not able to move the affected eye toward the "on" visual hemifield. Also, the maximum range was reduced. ATD transection produced similar deficits, but to a lesser extent. Alterations were also observed during vestibularly induced eye movements. As described previously (de la Cruz et al., 2000), MLF sectioning induced deficits in the amplitude of compensatory saccades and in the gain of the vestibulo-ocular reflex. ATD deprivation induced similar but less pronounced results than those after MLF transection, despite the vestibular origin of the ATD. A possible explanation for this result could be that the ATD projection is quantitatively smaller than that of the MLF (Baker and Highstein, 1978; Carpenter and Carleton, 1983; present data).

Partial deafferentation produced a decreased firing rate and a reduction in spontaneous and vestibular signals, which were comparable to those described for abducens internuclear and motoneurons after axotomy (Delgado-Garcia et al., 1988; de la Cruz et al., 2000; Davis-López de Carrizosa et al., 2009). In fact, axotomy leads to a retrograde synaptic stripping (Pastor et al., 2000), which implies a decrease in eye-related signals in abducens neurons (de la Cruz et al., 2000). Whereas axotomy produces a general afferent stripping, the selective removal by deafferentation results in specific afferent stripping. However, given the similarity of physiological results with axotomy, it should be concluded that the MLF input is crucial for the firing of medial rectus motoneurons and is not replaceable by the ATD input.

In the short term, the effects of ATD or MLF transection on motoneuron firing pattern were similar. In agreement with our results, ATD has been described as carrying not only head velocity information but also eye position signal (although weak), and, thus, the vestibular and MLF pathways would carry similar information, which might be considered redundant (Furuya and Markham, 1981; Reisine et al., 1981). A different hypothesis points to an additive action of both inputs, so that both of them would be necessary to properly activate medial rectus motoneurons (Baker and Highstein, 1978). In addition, based on the different distri- 
bution of vestibular (somatic) and abducens (dendritic) terminals on medial rectus motoneurons (Nguyen et al., 1999), vestibular inputs have been suggested to play a role in the balance of motoneuron excitability, which would serve as a control gate for MLF signals. Our results confirmed MLF/ATD terminal distribution, and, although motoneuron firing parameters decreased after both types of lesioning, it should be emphasized that MLF lesions yielded more pronounced changes than did ATD sections, suggesting a more relevant role for the MLF pathway but, also, that ATD is not functionally redundant, but it could be devised as a more primitive and default circuit subserving a basic vestibular role that is overlapped by the MLF pathway.

Long-term effects of ATD/MLF sections on medial rectus motoneuron discharge properties and eye movements

Long-term recordings of eye movements and firing rates of medial rectus motoneurons after MLF section revealed absence of significant recovery with time. In contrast, ATD animals exhibited a complete recovery of eye movements, which was accompanied by an equally complete recovery in motoneuron discharge characteristics during both spontaneous and vestibularly induced eye movements. To understand the possible mechanisms mediating this recovery, we performed morphological analysis of medial rectus motoneuron innervation. As stated above, Nguyen et al. (1999) postulated that ATD input to medial rectus motoneurons project mainly onto somata and proximal dendrites, while MLF inputs innervate more profusely distal dendrites. Our results are in agreement with this hypothesis, since (1) we observed a higher decrease in neuropil innervation after MLF than after ATD transection, and (2) although we did not detect differences in synaptic coverage around soma between long-term ATD and MLF animals, by $3 \mathrm{~d}$ postlesion there was a larger reduction in somatic innervation after ATD lesioning than after MLF lesioning. This result implies, on one hand, that, indeed, ATD axons end preferentially on motoneuron somatic membrane and, on the other hand, that there must be a compensatory mechanism after ATD depletion that allows the replacement of lost synaptic inputs. This hypothesis would fit with motor and neurophysiological data.

Sprouting after partial deafferentation has been described previously (Lund and Lund, 1971; Matthews et al., 1976; Lee et al., 1977; Masliah et al., 1991; Bäurle et al., 1992; Zhang et al., 1995). In our experiments, the most likely source of sprouting axons arose from the abducens internuclear neurons. This assumption would correlate well with the observed increase in calretinin staining after long-term ATD sectioning.

However, in addition to the formation of new terminals, the idea cannot be discarded that other plastic processes, such as increased synaptic efficacy between abducens internuclear neurons and medial rectus motoneurons, could have been involved in functional recovery, as described in deafferented vestibular neurons (Him and Dutia, 2001).

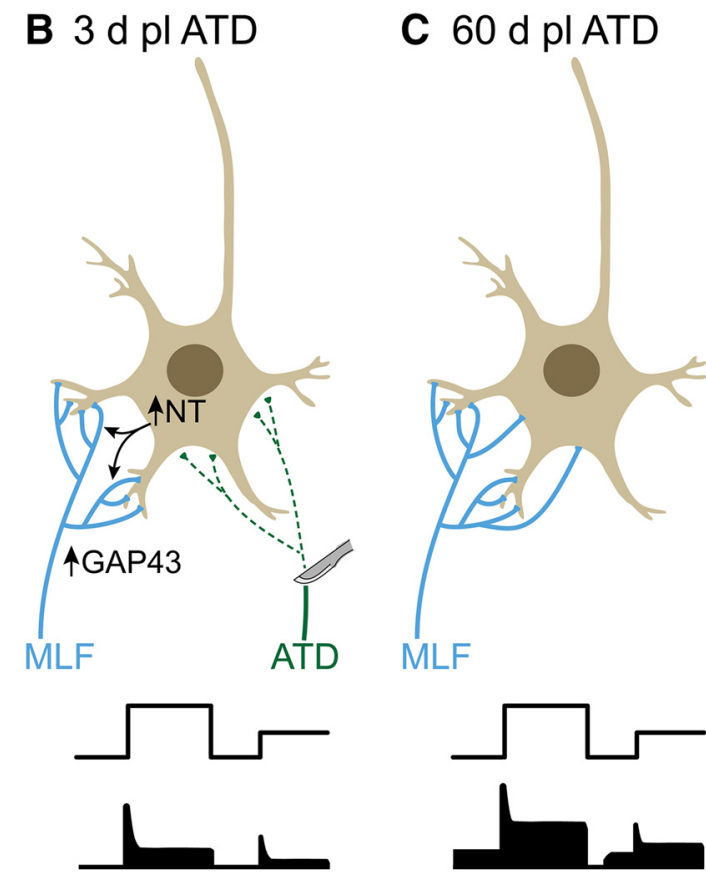

Figure 10. A, Schematic representation of medial rectus motoneurons innervated by MLF and ATD inputs in the control hight produce, in turn, axonal sprouting in this pathway and reinnervation of the partially deafferented motoneurons $(60 \mathrm{~d} \mathrm{pl} \mathrm{ATD,} 60 \mathrm{dpl}$ ATD), and thus the recovery of the normal firing pattern.

The same compensatory mechanisms could take place after MLF transection. However, the effects of this type of lesion were permanent, since 2 months postlesion all firing parameters were lower than those in control cells. We hypothesize that the ATD projection, with a few axons, is not sufficient to replace the information lost after the axotomy of the much larger projection of abducens internuclear neurons.

The severance of the MLF induces the internuclear ophthalmoplegia syndrome without preventing vergence (Gamlin et al., $1989 b$ ). The MLF comprises not only the ascending abducens internuclear neurons but the descending oculomotor internuclear neurons. As reported previously, neither the abducens internuclear neurons (Gamlin et al., 1989a) nor the oculomotor internuclear neurons (Clendaniel and Mays, 1994) carry appropriate signals for vergence. It is interesting to note that the severance of the oculomotor internuclear neurons does not produce ophthalmoplegia of the contralateral eye. Therefore, this projection should be comparably smaller than that of the abducens internuclear neurons.

ATD neurons could support firing of medial rectus motoneurons during convergence because they support an excitatory and ipsilateral projection to these motoneurons. However, the eye position sensitivity of ATD neurons makes them insufficient to support vergence (Reisine and Highstein, 1979). Other mesencephalic neurons projecting to the oculomotor nucleus and carrying signals related to both accommodation and/or vergence are the most appropriate neurons to drive vergence eye movements (Zhang et al., 1992).

\section{Possible molecular mechanisms for medial rectus motoneuron reinnervation}

Axonal sprouting is accompanied by an increase in GAP-43, a growth-associated protein whose expression is increased during 
axon elongation (Lynch et al., 1976; Benowitz et al., 1990; Lin et al., 1992; Gómez-Pinilla et al., 2004). The present results showed an increase in GAP-43 in the neuropil of medial rectus motoneuron subdivision $3 \mathrm{~d}$ after partial deafferentation, a period in which the physiological properties of the affected motoneurons were still altered. However, 2 months postlesion, GAP-43 levels were similar to those of the control side, in coincidence with partial (MLF) or complete (ATD) recovery. We postulate that, in the short term, remaining axons might be elongating their terminals covering the gaps left by a lesion, but new synapses would have not been established yet, in accordance with physiological results. However, in the long term, synapses would already be functional and GAP-43 downregulated, which would explain the recovery in motoneuron function (Fig. 10).

The molecular mechanisms by which GAP-43 was elevated in response to partial deafferentation could be linked to the increase in neurotrophin content in medial rectus motoneurons. In fact, exogenous NGF and NT-3 have been proven to increase sprouting after partial deafferentation in spinal cord motoneurons (Scott et al., 2005), and BDNF administration induces sprouting of spinal axons after spinal cord crush (Jakeman et al., 1998). Moreover, it has been proposed that NGF, BDNF, and NT-3 regulate GAP-43 content in growing axons (Perrone-Bizzozero et al., 1991, 1993; Mohiuddin et al., 1995; Federoff et al., 1988; Dinocourt et al., 2006; Geremia et al., 2010; Sanna et al., 2017) and that BDNF and NT-3 levels increase in spinal motoneurons after partial deafferentation (Johnson et al., 2000). Thus, considering that, first, the motoneuronal neurotrophin content was higher $3 \mathrm{~d}$ after partial deafferentation and, second, that both abducens internuclear and lateral vestibular neurons express the high-affinity neurotrophin receptors (Benítez-Temiño et al., 2004), it could be possible that neurotrophins released by motoneurons would be causally related to GAP-43-mediated sprouting (Fig. 10).

In summary, the major difference found between ATD and MLF lesions was the long-term complete recovery occurring after ATD transection, which was not present after MLF sectioning, and that could be explained by the regrowing of intact axons likely from abducens internuclear neurons.

\section{References}

Baizer JS, Baker JF (2005) Immunoreactivity for calcium-binding proteins defines subregions of the vestibular nuclear complex of the cat. Exp Brain Res 164:78-91. CrossRef Medline

Baker R, Highstein SM (1978) Vestibular projections to medial rectus subdivision of oculomotor nucleus. J Neurophysiol 41:1629-1646. Medline

Bäurle J, Grover BG, Grüsser-Cornehls U (1992) Plasticity of GABAergic terminals in Deiters' nucleus of weaver mutant and normal mice: a quantitative light microscope study. Brain Res 591:305-318. CrossRef Medline

Benítez-Temiño B, Morcuende S, Mentis GZ, de la Cruz RR, Pastor AM (2004) Expression of Trk receptors in the oculomotor system of the adult cat. J Comp Neurol 473:538-552. CrossRef Medline

Benowitz LI, Rodriguez WR, Neve RL (1990) The pattern of GAP-43 immunostaining changes in the rat hippocampal formation during reactive synaptogenesis. Brain Res Mol Brain Res 8:17-23. CrossRef Medline

Bienfang DC (1978) The course of direct projections from the abducens nucleus to the contralateral medial rectus subdivision of the oculomotor nucleus in the cat. Brain Res 145:277-289. CrossRef Medline

Büttner-Ennever JA (2006) The extraocular motor nuclei: organization and functional neuroanatomy. In: Neuroanatomy of the oculomotor system: progress in brain research, Vol 151 (Büttner-Ennever JA, ed), pp 95-126. Amsterdam: Elsevier.

Carpenter MB, Carleton SC (1983) Comparison of vestibular and abducens internuclear projections to the medial rectus subdivision of the oculomotor nucleus in the monkey. Brain Res 274:144-149. CrossRef Medline

Carpenter MB, McMasters RE (1963) Disturbances of conjugate horizontal eye movements in the monkey. II. Physiological effects and anatomical degeneration resulting from lesions in the medial longitudinal fasciculus. Arch Neurol 8:17-38.

Clendaniel RA, Mays LE (1994) Characteristics of antidromically identified oculomotor internuclear neurons during vergence and versional eye movements. J Neurophysiol 71:1111-1127. Medline

Davis-López de Carrizosa MA, Morado-Díaz CJ, Tena JJ, Benítez-Temiño B, Pecero ML, Morcuende SR, de la Cruz RR, Pastor AM (2009) Complementary actions of BDNF and neurotrophin-3 on the firing patterns and synaptic composition of motoneurons. J Neurosci 29:575-587. CrossRef Medline

de la Cruz RR, Escudero M, Delgado-García JM (1989) Behaviour of medial rectus motoneurons in the alert cat. Eur J Neurosci 1:288-295. CrossRef Medline

de la Cruz RR, Pastor AM, Martińez-Guijarro FJ, López-García C, DelgadoGarcía JM (1998) Localization of parvalbumin, calretinin, and calbindin D-28k in identified extraocular motoneurons and internuclear neurons of the cat. J Comp Neurol 390:377-391. CrossRef Medline

de la Cruz RR, Delgado-García JM, Pastor AM (2000) Discharge characteristics of axotomized abducens internuclear neurons in the adult cat. J Comp Neurol 427:391-404. CrossRef Medline

Delgado-Garcia JM, del Pozo F, Baker R (1986a) Behavior of neurons in the abducens nucleus of the alert cat-I. Motoneurons. Neuroscience 17: 929-952. Medline

Delgado-Garcia JM, del Pozo F, Baker R (1986b) Behavior of neurons in the abducens nucleus of the alert cat-II. Internuclear neurons. Neuroscience 17:953-973. Medline

Delgado-Garcia JM, Del Pozo F, Spencer RF, Baker R (1988) Behavior of neurons in the abducens nucleus of the alert cat-III. Axotomized motoneurons. Neuroscience 24:143-160. CrossRef Medline

Dinocourt C, Gallagher SE, Thompson SM (2006) Injury-induced axonal prouting in the hippocampus is initiated by activation of trkB receptors. Eur J Neurosci 24:1857-1866. CrossRef Medline

Escudero M, Delgado-García JM (1988) Behavior of reticular, vestibular and prepositus neurons terminating in the abducens nucleus of the alert cat. Exp Brain Res 71:218-222. Medline

Federoff HJ, Grabczyk E, Fishman MC (1988) Dual regulation of GAP-43 gene expression by nerve growth factor and glucocorticoids. J Biol Chem 263:19290-19295. Medline

Fuchs AF, Robinson DA (1966) A method for measuring horizontal and vertical eye movement chronically in the monkey. J Appl Physiol 21: 1068-1070. Medline

Fuchs AF, Scudder CA, Kaneko CR (1988) Discharge patterns and recruitment order of identified motoneurons and internuclear neurons in the monkey abducens nucleus. J Neurophysiol 60:1874-1895. Medline

Furuya N, Markham CH (1981) Arborization of axons in oculomotor nucleus identified by vestibular stimulation and intra-axonal injection of horseradish peroxidase. Exp Brain Res 43:289-303. Medline

Gamlin PD, Gnadt JW, Mays LE (1989a) Abducens internuclear neurons carry an inappropriate signal for ocular convergence. J Neurophysiol 62: 70-81. Medline

Gamlin PD, Gnadt JW, Mays LE (1989b) Lidocaine-induced unilateral internuclear ophthalmoplegia: effects on convergence and conjugate eye movements. J Neurophysiol 62:82-95. Medline

Geremia NM, Pettersson LM, Hasmatali JC, Hryciw T, Danielsen N, Schreyer DJ, Verge VM (2010) Endogenous BDNF regulates induction of intrinsic neuronal growth programs in injured sensory neurons. Exp Neurol 223:128-142. CrossRef Medline

Gómez-Pinilla F, Ying Z, Roy RR, Hodgson J, Edgerton VR (2004) Afferent input modulates neurotrophins and synaptic plasticity in the spinal cord. J Neurophysiol 92:3423-3432. CrossRef Medline

González-Forero D, Morcuende S, Alvarez FJ, de la Cruz RR, Pastor AM (2005) Transynaptic effects of tetanus neutoxin in the oculomotor system. Brain 128:2175-2188. CrossRef Medline

Grantyn R, Baker R, Grantyn A (1980) Morphological and physiological identification of excitatory pontine reticular neurons projecting to the cat abducens nucleus and spinal cord. Brain Res 198:221-228. CrossRef Medline

Highstein SM (1977) Abducens to medial rectus pathway in the MLF: a possible cellular basis for the syndrome of internuclear ophthalmoplegia. In: Eye movements (Brooks BA, Bajandas FJ, eds), pp 127-143. New York: Plenum.

Highstein SM, Baker R (1978) Excitatory termination of abducens internu- 
clear neurons on medial rectus motoneurons: relationship to syndrome of internuclear ophthalmoplegia. J Neurophysiol 41:1647-1661. Medline

Highstein SM, Holstein GR (2006) The anatomy of the vestibular nuclei. In: Neuroanatomy of the oculomotor system: progress in brain research, Vol 151 (Büttner-Ennever JA, ed), pp 157-203. Amsterdam: Elsevier.

Hikosaka O, Igusa Y, Nakao S, Shimazu H (1978) Direct inhibitory synaptic linkage of pontomedullary reticular burst neurons with abducens motoneurons in the cat. Exp Brain Res 33:337-352. Medline

Him A, Dutia MB (2001) Intrinsic excitability changes in vestibular nucleus neurons after unilateral deafferentation. Brain Res 908:58-66. CrossRef Medline

Igusa Y, Sasaki S, Shimazu H (1980) Excitatory premotor burst neurons in the cat pontine reticular formation related to the quick phase of vestibular nystagmus. Brain Res 182:451-456. CrossRef Medline

Jakeman LB, Wei P, Guan Z, Stokes BT (1998) Brain-derived neurotrophic factor stimulates hindlimb stepping and sprouting of cholinergic fibers after spinal cord injury. Exp Neurol 154:170-184. CrossRef Medline

Johnson RA, Okragly AJ, Haak-Frendscho M, Mitchell GS (2000) Cervical dorsal rhizotomy increases brain-derived neurotrophic factor and neurotrophin-3 expression in the ventral spinal cord. J Neurosci 20:RC77. Medline

Lee KS, Stanford EJ, Cotman CW, Lynch GS (1977) Ultrastructural evidence for bouton proliferation in the partially deafferented dentate gyrus of the adult rat. Exp Brain Res 29:475-485. Medline

Lin LH, Bock S, Carpenter K, Rose M, Norden JJ (1992) Synthesis and transport of GAP-43 in entorhinal cortex neurons and perforant pathway during lesion-induced sprouting and reactive synaptogenesis. Brain Res Mol Brain Res 14:147-153. CrossRef Medline

Lund RD, Lund JS (1971) Modifications of synaptic patterns in the superior colliculus of the rat during development and following deafferentation. Vision Res [Suppl 3]:281-298. Medline

Lynch G, Gall C, Rose G, Cotman C (1976) Changes in the distribution of the dentate gyrus associational system following unilateral or bilateral entorhinal lesions in the adult rat. Brain Res 110:57-71. CrossRef Medline

Masliah E, Fagan AM, Terry RD, DeTeresa R, Mallory M, Gage FH (1991) Reactive synaptogenesis assessed by synaptophysin immunoreactivity is associated with GAP-43 in the dentate gyrus of the adult rat. Exp Neurol 113:131-142. CrossRef Medline

Matthews DA, Cotman C, Lynch G (1976) An electron microscopic study of lesion-induced synaptogenesis in the dentate gyrus of the adult rat. II. Reappearance of morphologically normal synaptic contacts. Brain Res 115:23-41. CrossRef Medline

McCrea RA, Baker R (1985) Anatomical connections of the nucleus prepositus of the cat. J Comp Neurol 237:377-407. CrossRef Medline

McCrea RA, Yoshida K, Berthoz A, Baker R (1980) Eye movement related activity and morphology of second order vestibular neurons terminating in the cat abducens nucleus. Exp Brain Res 40:468-473. Medline
McCrea RA, Strassman A, Highstein SM (1986) Morphology and physiology of abducens motoneurons and internuclear neurons intracellularly injected with horseradish peroxidase in alert squirrel monkeys. J Comp Neurol 243:291-308. CrossRef Medline

Mohiuddin L, Fernandez K, Tomlinson DR, Fernyhough P (1995) Nerve growth factor and neurotrophin-3 enhance neurite outgrowth and upregulate the levels of messenger RNA for growth-associated protein GAP-43 and Tal a-tubulin in cultured adult rat sensory neurons. Neurosci Lett 185:20-23. CrossRef Medline

Nguyen LT, Baker R, Spencer RF (1999) Abducens internuclear and ascending tract of Deiters inputs to medial rectus motoneurons in the cat oculomotor nucleus: synaptic organization. J Comp Neurol 405:141-159. CrossRef Medline

Pastor AM, Torres B, Delgado-Garcia JM, Baker R (1991) Discharge characteristics of medial rectus and abducens motoneurons in the goldfish. J Neurophysiol 66:2125-2140. Medline

Pastor AM, Delgado-García JM, Martínez-Guijarro FJ, López-García C, de La Cruz RR (2000) Response of abducens internuclear neurons to axotomy in the adult cat. J Comp Neurol 427:370-390. CrossRef Medline

Perrone-Bizzozero NI, Neve RL, Irwin N, Lewis S, Fischer I, Benowitz LI (1991) Post-transcriptional regulation of GAP-43 mRNA levels during neuronal differentiation and nerve regeneration. Mol Cell Neurosci 2:402-409. CrossRef Medline

Perrone-Bizzozero NI, Cansino VV, Kohn DT (1993) Posttranscriptional regulation of GAP-43 gene expression in PC12 cells through protein kinase C-dependent stabilization of the mRNA J Cell Biol 120:1263-1270. Medline

Reisine H, Highstein SM (1979) The ascending tract of Deiters conveys a head velocity signal to medial rectus motoneurons. Brain Res 170:172176. CrossRef Medline

Reisine H, Strassman A, Highstein SM (1981) Eye position and eye velocity signals are conveyed to medial rectus motoneurons in the alert cat by the ascending tract of Deiters. Brain Res 211:153-157. CrossRef Medline

Sanna MD, Ghelardini C, Galeotti N (2017) HuD-mediated distinct BDNF regulatory pathways promote regeneration after nerve injury. Brain Res 1659:55-63. CrossRef Medline

Scott AL, Borisoff JF, Ramer MS (2005) Deafferentation and neurotrophinmediated intraspinal sprouting: a central role for the p75 neurotrophin receptor. Eur J Neurosci 21:81-92. CrossRef Medline

Zhang B, Goldberger ME, Wu LF, Murray M (1995) Plasticity of complex terminals in lamina II in partially deafferented spinal cord: the cat spared root preparation. Exp Neurol 132:186-193. CrossRef Medline

Zhang Y, Mays LE, Gamlin PD (1992) Characteristics of near response cells projecting to the oculomotor nucleus. J Neurophysiol 67:944-960. Medline 